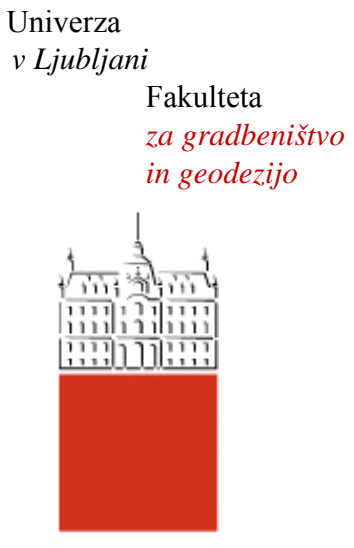

Jamova cesta 2

1000 Ljubljana, Slovenija

http://www3.fgg.uni-lj.si/

\section{DRUGG - Digitalni repozitorij UL FGG http://drugg.fgg.uni-lj.si/}

Ta članek je avtorjeva zadnja recenzirana različica, kot je bila sprejeta po opravljeni recenziji.

Prosimo, da se pri navajanju sklicujte na bibliografske podatke, kot je navedeno:
University
of Ljubljana

Faculty of

Civil and Geodetic

Engineering

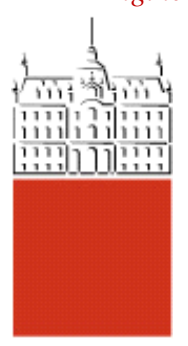

Jamova cesta 2

SI - 1000 Ljubljana, Slovenia

http://www3.fgg.uni-lj.si/en/

DRUGG - The Digital Repository http://drugg.fgg.uni-lj.si/

This version of the article is author's manuscript as accepted for publishing after the review process.

When citing, please refer to the publisher's bibliographic information as follows:

Bratina, S., Saje, M., Planinc, I. 2004. On materially and geometrically non-linear analysis of reinforced concrete planar frames. International Journal of Solids and Structures 41,24-25: 7181-7207. DOI: 10.1016/j.ijsolstr.2004.06.004. 


\title{
On materially and geometrically non-linear analysis of reinforced concrete planar frames
}

\author{
S. Bratina, M. Saje ${ }^{1}$ and I. Planinc \\ University of Ljubljana, Faculty of Civil and Geodetic Engineering \\ Jamova 2, SI-1115 Ljubljana, Slovenia
}

\begin{abstract}
A family of new beam finite elements for geometrically and materially non-linear static analysis of reinforced concrete planar frames is derived, in which strain measures are the only interpolated unknowns, and where the constitutive and equilibrium internal forces are equal at integration points. The strain-localization caused by the strain-softening at cross-sections is resolved by the introduction of a 'short constant-strain element'. Comparisons between numerical and experimental results on planar frames in pre- and post-critical states show both good accuracy and computational efficiency of the present formulation.
\end{abstract}

Keywords: reinforced concrete frame; strain-based finite element; strain softening; localization; Reissner's beam theory.

\section{Introduction}

Since it was reinforced by steel bars more than a century ago, concrete has been a very suitable constructional material, and reinforced concrete structures have played an important role in the built environment. For obvious reasons, computational models for the everyday structural analysis of reinforced concrete structures were simple and conservative at first. Nowadays, when the capabilities of the mechanical modelling are much larger, an engineer can perform analyses which give considerably better predictions of stresses,

\footnotetext{
${ }^{1}$ Corresponding author. Phone.: +386 14768 613; fax: +386 14768629 .

E-mail address: msaje@fgg.uni-lj.si (M. Saje)
} 
displacements, limit loads and mechanisms of the damage and the destruction. There are, however, several problems that still need to be solved if we want to predict the behaviour of reinforced concrete structures even more reliably and with a greater precision. One such problem is the softening of concrete and its effect on the mechanical response of structures.

The accuracy of the non-linear analysis of a reinforced concrete structure largely depends on how we model concrete, steel, and their mutual interaction, and which method of the discretization we use. The behaviour of concrete and steel under various loading conditions has been experimentally studied for long and is well described; the mathematical description of the concrete behaviour, on the other hand, is still not sufficiently developed (see Contrafatto and Cuomo, 2002; and Grassel et al., 2002, among others, for their new constitutive models and comments on their behaviour). A number of discretization methods have so far been proposed, many of them with the particular emphasis on the effect of softening of concrete on the global (often softening) response of the structure (see Chen et al., 2000; Peerlings et al., 2002; or Wells and Sluys, 2000).

Engineering structures are often composed of beams, plates and shells, and have complicated geometrical shapes. At the present state of the computer hardware, the full 3D non-linear structural analysis of structures is very limited (Khaloo and Tariverdilo, 2002). That is why we usually employ $1 \mathrm{D}$ (beam) and 2D (plate and shell) finite elements in the structural analysis and why the research to improve 1D and 2D finite elements is still continuing.

One of the essential steps in the beam modelling is the way material is considered. There are two choices available. We can either assume the constitutive equations of the crosssection, which relate its stress-resultants to cross-sectional deformation variables (Simo et al., 1984; Kwak and Kim, 2002) - the applicability of this kind of constitutive equations is simple if rather limited, or we can assume the constitutive equations of a fibre, which relate its stress and strain. While the latter is a much more accurate approach, making it possible to include various material phenomena, it is computationally more demanding as it needs the integration of stresses and material moduli across the cross-section at each Gaussian point of an element and at each Newton's iteration.

The topic of the strain-softening in brittle heterogeneous materials has been widely discussed for years, see, e.g. Bažant et al. (1987a, 1987b), and Maier and Perego (1992), particularly with regard to the deduction of efficient numerical procedures (Bažant and 
Mazars, 1990). In the context of concrete frame-like structures, two approaches have been suggested. The most important assumption of the first one is that the strain-softening triggers the point-wise strain localization (a 'plastic hinge'); once the hinge emerges, its subsequent behaviour is governed by the moment-rotation law obtained experimentally (Jirásek, 1997). This model is simple to use and independent of the finite-element mesh, but it disregards the effect of extensional strain and is thus convenient only for a certain class of problems. The second approach is based on experimental evidence that the softening of material develops on a finite-length region of a concrete beam (Bažant et al., 1987a, 1987b). The present paper follows the second approach.

The literature on geometrically linear-materially non-linear analysis of reinforced concrete frames is extensive, see, e.g. the works by Bažant et al. (1987a, 1987b), Kim and Lee (1992), Lazaro and Richards (1973), Pöttier and Swoboda (1987), Darvall and Mendis (1985), or Rasheed and Dinno (1994), among many, and the references therein. In contrast, a lot less publications can be found which consider both geometric and material nonlinearity, see, e.g. the paper by El-Metwally et al. (1990) on the stability of a reinforced concrete column, or Carol and Murcia (1989), Gunnin et al. (1989), and Mendis and Darvall (1988).

In the present paper we deal with the fibre-based constitutive equations of concrete and steel, and employ standard non-linear material laws, as used in design, but disregard the phenomena such as creep and shrinkage of concrete. Our main goal is to show a new approach to the strain-softening analysis of reinforced concrete frame-like structures. We derive a family of new beam finite elements for the geometrically and materially nonlinear static analysis of reinforced concrete planar frames. The kinematically exact beam theory according to Reissner (1972) is employed as a theoretical basis of our finite-element formulation. There are two original contributions in our finite elements: (i) strains are the only interpolated unknowns; (ii) the constitutive and equilibrium internal forces are enforced to be equal at integration points. While one of the consequences of the former is the absence of locking, the latter assures the equality of the bearing capacity of the chosen cross-sections and their stress-resultants due to the imposed loading.

While the effect of shear strain is considered in our theoretical deductions, it is disregarded in our numerical examples. The reinforcing steel bars are modelled as steel layers within a generally inhomogeneous, layered, rigid cross-section where the conformity of axial 
strains in concrete and in steel layers at the contact is assumed. The behaviour of concrete in compression and tension is modelled and analysed by the constitutive laws of Eurocode 2 (1999) and Desayi and Krishnan (1964). Both models exhibit the strain-softening in compression. The behaviour of steel reinforcing bars is assumed to be elasto-plastic with a strain-softening branch. What regards the strain-softening and strain-localization, the essential step in our formulation is the introduction of a 'short constant-strain element' (as was in the context of the geometrically linear theory suggested by Bažant et al., 1987a; Darvall and Mendis, 1985; Kim and Lee, 1992; Coleman and Spacone, 2001).

\section{Formulation of the non-linear planar beam model}

\subsection{Kinematic relations}

We consider a planar beam of initial, undeformed length $L$, and constant reinforced concrete cross-section $A$. The deformed configuration of the beam is described by a planar curve - the line of centroids of the concrete section, and by the family of its cross-sections. The relative position of a centroidal particle with respect to the curve is identified by the material coordinate, $x \in[0, L]$. Its absolute position with respect to the spatial Cartesian coordinate system with coordinates $X, Y, Z$, and unit base vectors $\boldsymbol{E}_{X}, \boldsymbol{E}_{Y}, \boldsymbol{E}_{Z}$, is given by vector $\boldsymbol{R}_{0}(x)$. The cross-sectional plane at $x$ is identified by the unit vector $\boldsymbol{e}_{x}(x)$ perpendicular to the cross-section, and by the in-plane unit vectors $\boldsymbol{e}_{y}(x)$ and $\boldsymbol{e}_{z}(x)$ with $\boldsymbol{e}_{y}(x)=\boldsymbol{E}_{Y}$. Vectors $\boldsymbol{e}_{x}(x), \boldsymbol{e}_{y}(x), \boldsymbol{e}_{z}(x)$ constitute the basis of material coordinate system with material coordinates $x, y, z$. An arbitrary particle of the cross-sectional plane at $x$ is identified by a pair of material coordinates, $(y, z) \in A$. An arbitrary particle of the beam is identified by a trio of coordinates, $(x, y, z) \in L \times A$. The deformed position of particle $(x, y, z)$ with respect to the spatial coordinate system is given by

$$
\boldsymbol{R}(x, y, z)=\boldsymbol{R}_{0}(x)+y \boldsymbol{e}_{y}(x)+z \boldsymbol{e}_{z}(x)=X(x, y, z) \boldsymbol{E}_{X}+Y(x, y, z) \boldsymbol{E}_{Y}+Z(x, y, z) \boldsymbol{E}_{Z} .
$$

We assume that the geometrical shape of the cross-section and the distribution of material (i.e. concrete and steel) are symmetric with respect to the plane of deformation, $(X, Z)$. For the simplicity of derivation, we assume that the beam in the undeformed configuration is straight and lies on $X$-axis of the spatial coordinate system so that the spatial and material coordinates coincide initially: $X=x, Y=y, Z=z$, and $\boldsymbol{E}_{X}=\boldsymbol{e}_{x}^{0}, \boldsymbol{E}_{Y}=\boldsymbol{e}_{y}^{0}$, 
$\boldsymbol{E}_{Z}=\boldsymbol{e}_{z}^{0}$. Consequently, in the undeformed configuration, Eqn (1) is reduced to

$$
\boldsymbol{r}(x, y, z)=\boldsymbol{r}_{0}(x)+y \boldsymbol{e}_{y}^{0}(x)+z \boldsymbol{e}_{z}^{0}(x)=x \boldsymbol{E}_{X}+y \boldsymbol{E}_{Y}+z \boldsymbol{E}_{Z}
$$

where $\boldsymbol{r}_{0}=x \boldsymbol{E}_{X}$ and $\boldsymbol{r}$ are position vectors of centroidal particle $(x, 0,0)$ and particle $(x, y, z)$, respectively, and $\boldsymbol{e}_{y}^{0}$ and $\boldsymbol{e}_{z}^{0}$ are the undeformed material base vectors. By introducing the displacement vector of the centroidal axis, $\boldsymbol{u}=\boldsymbol{R}_{0}-\boldsymbol{r}_{0}=u \boldsymbol{E}_{X}+v \boldsymbol{E}_{Y}+w \boldsymbol{E}_{Z}$, and the condition $v=0$ for the planar motion of the beam into Eqn (1), we obtain (see Fig. 1)

$$
\boldsymbol{R}(x, y, z)=\boldsymbol{R}_{0}(x)+y \boldsymbol{e}_{y}(x)+z \boldsymbol{e}_{z}(x)=(x+u(x)) \boldsymbol{E}_{X}+w(x) \boldsymbol{E}_{Z}+y \boldsymbol{e}_{y}(x)+z \boldsymbol{e}_{z}(x),
$$

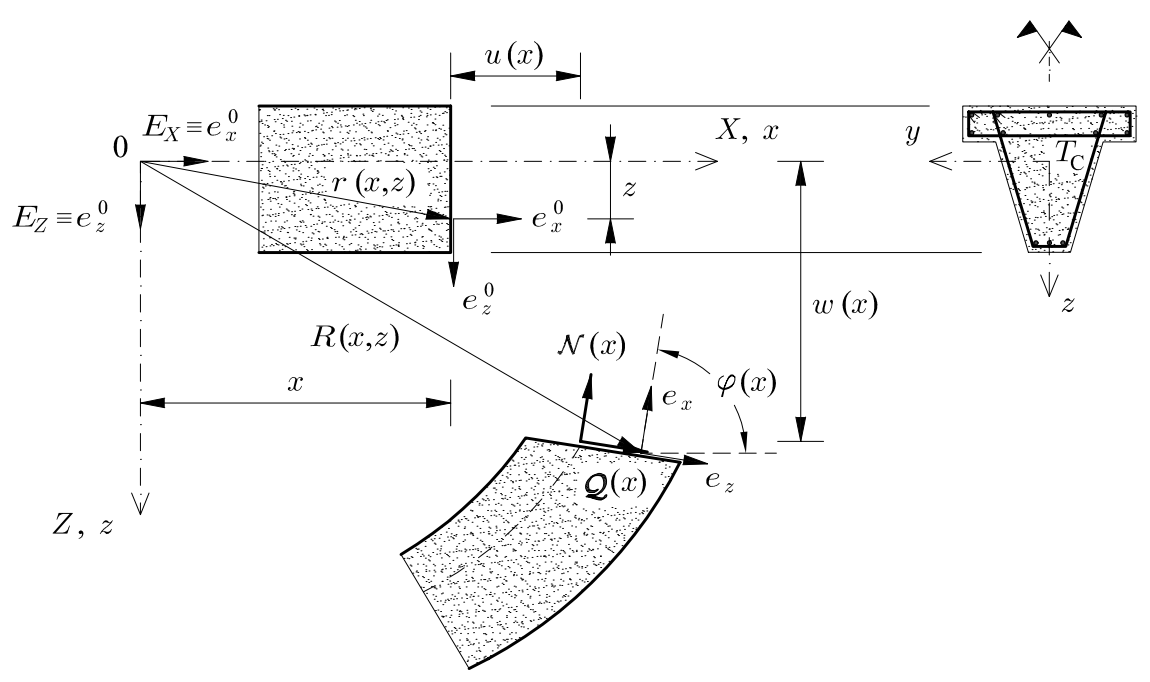

Figure 1: Deformed and undeformed configurations of the beam.

where $u$ and $w$ are the components of the displacement vector with respect to the spatial basis. They are related to the strain measures $\varepsilon, \gamma$, and $\kappa$ by the kinematic equations (Reissner, 1972)

$$
\begin{array}{r}
1+u^{\prime}(x)-(1+\varepsilon(x)) \cos \varphi(x)-\gamma(x) \sin \varphi(x)=0, \\
w^{\prime}(x)+(1+\varepsilon(x)) \sin \varphi(x)-\gamma(x) \cos \varphi(x)=0, \\
\varphi^{\prime}(x)-\kappa(x)=0 .
\end{array}
$$

Here, $\varepsilon(x)>-1$ is extensional strain of the axis; $\gamma(x)$ is its shear strain; $\kappa(x)$ is bending strain, closely related to the curvature of the axis (Vratanar and Saje, 1998); and $\varphi(x)$ is 
the rotation of the cross-section (Fig. 1). In (4)-(6), the prime $\left(^{\prime}\right)$ denotes the derivative with respect to $x$. As no bounds on the displacements and the rotation are set, the assumed kinematics is usually marked as kinematically or geometrically exact. $\varepsilon, \gamma$, and $\kappa$ are deformation variables, while $u, w$, and $\varphi$ are termed kinematic variables.

The extensional strain of an arbitrary particle $(x, y, z)$ will be denoted by $D(x, y, z)$. It is a linear function of $z$

$$
D(x, y, z)=\varepsilon(x)+z \kappa(x),
$$

which is the implication of the assumed Bernoulli hypothesis. It is clear from Eqn (7) that $D$ depends on $x$ and $z$ only. Hence, the notation $D(x, z)$ will be used.

\subsection{Equilibrium equations}

The equilibrium equations of the beam consist of three scalar differential equations of the first order (Saje, 1990)

$$
\begin{aligned}
\mathcal{R}_{1}^{\prime}(x)+p_{X}(x) & =0, \\
\mathcal{R}_{2}^{\prime}(x)+p_{Z}(x) & =0, \\
\mathcal{M}^{\prime}(x)+\left(1+u^{\prime}(x)\right) \mathcal{R}_{2}(x)-w^{\prime}(x) \mathcal{R}_{1}(x)+m_{Y}(x) & =0,
\end{aligned}
$$

for the three components $\mathcal{R}_{1}(x), \mathcal{R}_{2}(x)$, and $\mathcal{M}(x)$ of the cross-sectional stress-resultants, $\boldsymbol{N}(x)=\mathcal{R}_{1}(x) \boldsymbol{E}_{X}+\mathcal{R}_{2}(x) \boldsymbol{E}_{Z}$ and $\boldsymbol{M}(x)=\mathcal{M}(x) \boldsymbol{E}_{Y}$. In Eqns (8)-(10), $p_{X}(x), p_{Z}(x)$, and $m_{Y}(x)$ are the external distributed force and moment loads per unit of undeformed centroidal axis in the $X, Z$, and $Y$ directions, respectively. The stress-resultant $N$ can also be expressed in the material basis, i.e. $\boldsymbol{N}(x)=\mathcal{N}(x) \boldsymbol{e}_{x}+\mathcal{Q}(x) \boldsymbol{e}_{z}$. Its components, $\mathcal{N}$ and $\mathcal{Q}$, are axial and shear forces of the cross-section. They are related to $\mathcal{R}_{1}$ and $\mathcal{R}_{2}$ by (Saje, 1990)

$$
\begin{aligned}
\mathcal{N} & =\mathcal{R}_{1} \cos \varphi-\mathcal{R}_{2} \sin \varphi \\
\mathcal{Q} & =\mathcal{R}_{1} \sin \varphi+\mathcal{R}_{2} \cos \varphi
\end{aligned}
$$

$\mathcal{N}, \mathcal{Q}$, and $\mathcal{M}$ are called the equilibrium axial and shear force, and the equilibrium bending moment, respectively, in contrast to the constitutive forces and moment, which will be defined in the next section. 


\subsection{Constitutive relations}

The constitutive relations between the extensional strain, $D=\varepsilon+z \kappa$, and the normal stress, $\sigma$, and the shear strain, $\gamma$, and the shear stress, $\tau$, are assumed to be given by the formal relations

$$
\begin{gathered}
\sigma=\mathcal{F}(D), \\
\tau=\mathcal{G}(\gamma) .
\end{gathered}
$$

Functions $\mathcal{F}(D)$ and $\mathcal{G}(\gamma)$ are chosen according to a specific material to be modelled. In reinforced concrete beams, the effect of shear is usually small compared to the effects of bending, compression and tension, and is hard to be described mathematically. We will assume a simple linear constitutive equation $\mathcal{G}(\gamma)=G \gamma$, where $G$ is shear modulus of material. The integration of stresses over the cross-section yields the so called constitutive axial and shear forces and the constitutive moment:

$$
\begin{array}{r}
\mathcal{N}_{\mathrm{c}}(\varepsilon, \kappa)=\int_{A} \sigma \mathrm{d} A=\int_{A} \mathcal{F}(D) \mathrm{d} A, \\
\mathcal{Q}_{\mathrm{c}}(\gamma)=\int_{A} \tau \mathrm{d} A=\int_{A} \mathcal{G}(\gamma) \mathrm{d} A=G A_{\mathrm{s}} \gamma(x), \\
\mathcal{M}_{\mathrm{c}}(\varepsilon, \kappa)=\int_{A} z \sigma \mathrm{d} A=\int_{A} z \mathcal{F}(D) \mathrm{d} A .
\end{array}
$$

$A_{\mathrm{s}}<A$ is the shear area of the cross-section (Cowper, 1966). The equilibrium requires the constitutive and equilibrium forces and moments at cross-sections to be equal, $\mathcal{N}_{\mathrm{c}}=\mathcal{N}$, $\mathcal{Q}_{\mathrm{c}}=\mathcal{Q}$, and $\mathcal{M}_{\mathrm{c}}=\mathcal{M}$, or equivalently,

$$
\begin{aligned}
\mathcal{N}_{\mathrm{c}}(\varepsilon(x), \kappa(x))-\mathcal{N}(x) & =0, \\
\mathcal{Q}_{\mathrm{c}}(\gamma(x))-\mathcal{Q}(x) & =0, \\
\mathcal{M}_{\mathrm{c}}(\varepsilon(x), \kappa(x))-\mathcal{M}(x) & =0 .
\end{aligned}
$$

In the application of Newton's method for the solution of discrete equations of the beam, we need the variations of $\mathcal{N}_{\mathrm{c}}, \mathcal{Q}_{\mathrm{c}}$, and $\mathcal{M}_{\mathrm{c}}$ with respect to $\varepsilon, \gamma$, and $\kappa$. Varying Eqns (15)-(17) yields

$$
\begin{array}{r}
\delta \mathcal{N}_{\mathrm{c}}=\left(\int_{A} \frac{\partial \sigma}{\partial D} \mathrm{~d} A\right) \delta \varepsilon+\left(\int_{A} z \frac{\partial \sigma}{\partial D} \mathrm{~d} A\right) \delta \kappa=C_{11} \delta \varepsilon+C_{13} \delta \kappa \\
\delta \mathcal{Q}_{\mathrm{c}}=\left(\int_{A} \frac{\partial \tau}{\partial \gamma} \mathrm{d} A\right) \delta \gamma=C_{22} \delta \gamma, \\
\delta \mathcal{M}_{\mathrm{c}}=\left(\int_{A} z \frac{\partial \sigma}{\partial D} \mathrm{~d} A\right) \delta \varepsilon+\left(\int_{A} z^{2} \frac{\partial \sigma}{\partial D} \mathrm{~d} A\right) \delta \kappa=C_{31} \delta \varepsilon+C_{33} \delta \kappa .
\end{array}
$$


Functions $C_{11}(x), C_{13}(x)=C_{31}(x), C_{33}(x)$, and $C_{22}(x)=G(x) A_{\mathrm{s}}$ are the components of the tangent constitutive matrix of the cross-section, $\boldsymbol{C}(x)$. They depend on the distribution of the tangent material moduli over the cross-section, and on its geometrical shape. For stable materials, $\boldsymbol{C}$ has to be positive definite for any $x$, i.e., its principal minors must be positive:

$$
C_{11}>0, \quad C_{11} C_{22}>0, \quad \operatorname{det} \boldsymbol{C}=C_{22}\left(C_{11} C_{33}-C_{13}^{2}\right)>0 .
$$

As soon as one of the conditions (24) is violated, the material instability takes place in the cross-section. Materials, which do not fulfil the conditions in Eqn (24), are unstable materials. Concrete, when strained in the strain-softening regime, is unstable material.

\subsection{Principle of virtual work}

The principle of virtual work states that the difference of virtual work of the internal and the external forces is zero (e.g. Reissner, 1972; Saje, 1990)

$$
\int_{0}^{L}(\mathcal{N} \delta \varepsilon+\mathcal{Q} \delta \gamma+\mathcal{M} \delta \kappa) \mathrm{d} x-\int_{0}^{L}\left(p_{X} \delta u+p_{Z} \delta w+m_{Y} \delta \varphi\right) \mathrm{d} x-\sum_{i=1}^{6} S_{i} \delta u_{i}=0
$$

In Eqn (25) $\delta u, \delta w$, and $\delta \varphi$ denote virtual displacements and rotation, $\delta \varepsilon, \delta \gamma$, and $\delta \kappa$ are virtual strains, and $\delta u_{i}(i=1,2, \ldots, 6)$ are virtual boundary displacements and rotations at end points of the beam:

$$
\delta u_{1}=\delta u(0), \delta u_{2}=\delta w(0), \delta u_{3}=\delta \varphi(0), \delta u_{4}=\delta u(L), \delta u_{5}=\delta w(L), \delta u_{6}=\delta \varphi(L)
$$

$S_{i}(i=1,2, \ldots, 6)$ are their work-complementary generalized boundary external loads.

\section{Finite element approximation and solution procedure}

\subsection{Modified principle of virtual work}

We assume that Eqns (18)-(20) are identically satisfied. Then $\mathcal{N}, \mathcal{Q}$, and $\mathcal{M}$ can be replaced by $\mathcal{N}_{\mathrm{c}}, \mathcal{Q}_{\mathrm{c}}$, and $\mathcal{M}_{\mathrm{c}}$ and the principle of virtual work expressed in Eqn (25) takes the form

$$
\int_{0}^{L}\left(\mathcal{N}_{\mathrm{c}} \delta \varepsilon+\mathcal{Q}_{\mathrm{c}} \delta \gamma+\mathcal{M}_{\mathrm{c}} \delta \kappa\right) \mathrm{d} x-\int_{0}^{L}\left(p_{X} \delta u+p_{Z} \delta w+m_{Y} \delta \varphi\right) \mathrm{d} x-\sum_{i=1}^{6} S_{i} \delta u_{i}=0 .
$$

The principle of virtual work assumes that the deformation and kinematic variables identically satisfy the kinematic equations (4)-(6). This means that only three out of six 
variables, $u, w, \varphi, \varepsilon, \gamma$, and $\kappa$, are independent. Often the three of them $(u, w, \varphi)$ are taken as independent. Then the functional in (26) becomes the function of three independent functions, $u, w$, and $\varphi$. Such formulations are used, among others, by Bathe (1996), Crisfield (1991), or Stolarski and Belytschko (1983). These formulations are prone to locking unless the reduced numerical integration is used. In the present paper, we follow a different approach by introducing a modified principle of virtual work, and derive finite elements in which the extensional strain, $\varepsilon(x)$, and the bending strain, $\kappa(x)$, are the only independent functions.

The principle of virtual work (26) is considered as the constrained variational principle in which the kinematic equations (4)-(6) play the role of constraints. According to the constrained minimization methodology, we introduce three Lagrangian multipliers $\mathcal{R}_{1}(x)$, $\mathcal{R}_{2}(x)$, and $\mathcal{M}(x)$, by which the constraining equations are multiplied and then integrated over the domain $[0, L]$. The resulting equations are varied with respect to now independent functions $u, w, \varphi, \varepsilon, \gamma, \kappa, \mathcal{R}_{1}, \mathcal{R}_{2}$, and $\mathcal{M}$. Integrals $\int_{0}^{L} \mathcal{R}_{1} \delta u^{\prime} \mathrm{d} x, \int_{0}^{L} \mathcal{R}_{2} \delta w^{\prime} \mathrm{d} x$ and $\int_{0}^{L} \mathcal{M} \delta \varphi^{\prime} \mathrm{d} x$ are integrated by parts. The equations thus derived are added to the principle (26). After rearranging terms we obtain an extended principle of virtual work:

$$
\begin{array}{r}
\int_{0}^{L}\left(\left(\mathcal{N}_{\mathrm{c}}-\mathcal{N}\right) \delta \varepsilon+\left(\mathcal{Q}_{\mathrm{c}}-\mathcal{Q}\right) \delta \gamma+\left(\mathcal{M}_{\mathrm{c}}-\mathcal{M}\right) \delta \kappa\right) \mathrm{d} x- \\
\int_{0}^{L}\left(\left(p_{X}+\mathcal{R}_{1}^{\prime}\right) \delta u-\left(p_{Z}+\mathcal{R}_{2}^{\prime}\right) \delta w-\left(\mathcal{M}^{\prime}-(1+\varepsilon) \mathcal{Q}+\gamma \mathcal{N}+m_{Y}\right) \delta \varphi\right) \mathrm{d} x+ \\
\int_{0}^{L}\left(1+u^{\prime}-(1+\varepsilon) \cos \varphi-\gamma \sin \varphi\right) \delta \mathcal{R}_{1} \mathrm{~d} x+ \\
\int_{0}^{L}\left(w^{\prime}+(1+\varepsilon) \sin \varphi-\gamma \cos \varphi\right) \delta \mathcal{R}_{2} \mathrm{~d} x+\int_{0}^{L}\left(\varphi^{\prime}-\kappa\right) \delta \mathcal{M} \mathrm{d} x- \\
\left(S_{1}+\mathcal{R}_{1}(0)\right) \delta u_{1}-\left(S_{2}+\mathcal{R}_{2}(0)\right) \delta u_{2}-\left(S_{3}+\mathcal{M}(0)\right) \delta u_{3}- \\
\left(S_{4}-\mathcal{R}_{1}(L)\right) \delta u_{4}-\left(S_{5}-\mathcal{R}_{2}(L)\right) \delta u_{5}-\left(S_{6}-\mathcal{M}(L)\right) \delta u_{6}=0 .
\end{array}
$$

In Eqn (27) the variations $\delta \varepsilon, \delta \gamma, \delta \kappa, \delta u, \delta w, \delta \varphi, \delta \mathcal{R}_{1}, \delta \mathcal{R}_{2}$, and $\delta \mathcal{M}$ are arbitrary independent functions, while the variations $\delta u_{1}=\delta u(0), \delta u_{2}=\delta w(0), \delta u_{3}=\delta \varphi(0), \delta u_{4}=\delta u(L)$, $\delta u_{5}=\delta w(L)$, and $\delta u_{6}=\delta \varphi(L)$ are arbitrary independent parameters. In accordance with the fundamental lemma of the calculus of variations, the coefficients at the independent variations should be zero, which gives the Euler-Lagrange equations of the principle. They are the constitutive equations (18)-(20), the kinematic equations (4)-(6), and the equilibrium equations (8)-(10). The related natural (or static) and essential (or kinematic) boundary conditions are: 


$$
\begin{array}{cccc}
S_{1}+\mathcal{R}_{1}(0)=0 & \text { or } & u(0)=u_{1}, \\
S_{2}+\mathcal{R}_{2}(0)=0 & \text { or } & w(0)=u_{2}, \\
S_{3}+\mathcal{M}(0)=0 & \text { or } & \varphi(0)=u_{3}, \\
S_{4}-\mathcal{R}_{1}(L)=0 & \text { or } & u(L)=u_{4}, \\
S_{5}-\mathcal{R}_{2}(L)=0 & \text { or } & w(L)=u_{5}, \\
S_{6}-\mathcal{M}(L)=0 & \text { or } & \varphi(L)=u_{6} .
\end{array}
$$

For a given loading factor, $\lambda$, the Euler-Lagrange equations constitute a system of nine non-linear algebraic-differential equations for nine unknown functions $\varepsilon(x), \gamma(x), \kappa(x)$, $u(x), w(x), \varphi(x), \mathcal{R}_{1}(x), \mathcal{R}_{2}(x)$, and $\mathcal{M}(x)$, subject to the set of boundary conditions (28)-(33). In order to minimize the number of unknown functions in our final variational principle, some of these equations are integrated separately.

The integration of Eqns (4)-(6) yields

$$
\begin{array}{r}
u(x)=u(0)+\int_{0}^{x}((1+\varepsilon) \cos \varphi+\gamma \sin \varphi) \mathrm{d} \xi-x, \\
w(x)=w(0)-\int_{0}^{x}((1+\varepsilon) \sin \varphi-\gamma \cos \varphi) \mathrm{d} \xi \\
\varphi(x)=\varphi(0)+\int_{0}^{x} \kappa \mathrm{d} \xi .
\end{array}
$$

It is clear from these equations that while $u(0), w(0), \varphi(0)$ can be arbitrary, parameters $u(L), w(L), \varphi(L)$ cannot meet arbitrary boundary conditions at $x=L$, unless $\varepsilon(x), \gamma(x)$, and $\kappa(x)$ explicitly satisfy the conditions

$$
\begin{array}{r}
u(L)=u(0)+\int_{0}^{L}((1+\varepsilon) \cos \varphi+\gamma \sin \varphi) \mathrm{d} x-L, \\
w(L)=w(0)-\int_{0}^{L}((1+\varepsilon) \sin \varphi-\gamma \cos \varphi) \mathrm{d} x, \\
\varphi(L)=\varphi(0)+\int_{0}^{L} \kappa \mathrm{d} x .
\end{array}
$$

The integration of Eqns (8)-(10) gives

$$
\begin{array}{r}
\mathcal{R}_{1}(x)=\mathcal{R}_{1}(0)-\int_{0}^{x} p_{X} \mathrm{~d} \xi \\
\mathcal{R}_{2}(x)=\mathcal{R}_{2}(0)-\int_{0}^{x} p_{Z} \mathrm{~d} \xi \\
\mathcal{M}(x)=\mathcal{M}(0)+\int_{0}^{x}\left((1+\varepsilon) \mathcal{Q}-\gamma \mathcal{N}-m_{Y}\right) \mathrm{d} \xi
\end{array}
$$


Similarly, it is clear that while $\mathcal{R}_{1}(0), \mathcal{R}_{2}(0)$, and $\mathcal{M}(0)$ can fulfil any natural boundary conditions at $x=0$, multipliers $\mathcal{R}_{1}(L), \mathcal{R}_{2}(L)$, and $\mathcal{M}(L)$ cannot, because they depend on the given deformation variables $\varepsilon(x), \gamma(x)$, and $\kappa(x)$. Therefore, in order to meet the prescribed natural boundary conditions at $x=L$, we must explicitly require that

$$
\begin{array}{r}
\mathcal{R}_{1}(L)=\mathcal{R}_{1}(0)-\int_{0}^{L} p_{X} \mathrm{~d} x, \\
\mathcal{R}_{2}(L)=\mathcal{R}_{2}(0)-\int_{0}^{L} p_{Z} \mathrm{~d} x, \\
\mathcal{M}(L)=\mathcal{M}(0)+\int_{0}^{L}\left((1+\varepsilon) \mathcal{Q}-\gamma \mathcal{N}-m_{Y}\right) \mathrm{d} x .
\end{array}
$$

Eqns (34)-(36) and (40)-(42) make it possible for the kinematic and static variables to be expressed with the deformation variables. When these relations are inserted into the extended principle of virtual work, Eqn (27), the terms multiplied by $\delta u, \delta w, \delta \varphi, \delta \mathcal{R}_{1}$, $\delta \mathcal{R}_{2}$, and $\delta \mathcal{M}$ vanish. Once Eqns (43)-(45) are considered in the principle (27), Eqns (37)-(39) added and the terms rearranged, we obtain the principle which depends solely on the deformation functions, $\varepsilon(x), \gamma(x), \kappa(x)$.

This principle is further modified by eliminating $\gamma$ with the help of Eqn (19). If $\partial \mathcal{Q}_{\mathrm{c}} / \partial \gamma=G A_{\mathrm{s}} \neq 0$, the linear equation (19) can uniquely be solved for $\gamma$, yielding

$$
\gamma=\bar{\gamma}\left(\kappa, \mathcal{R}_{1}(0), \mathcal{R}_{2}(0), \varphi(0)\right)=\frac{\mathcal{R}_{1} \sin \varphi+\mathcal{R}_{2} \cos \varphi}{G A_{\mathrm{S}}}=\frac{\mathcal{Q}}{G A_{\mathrm{s}}}
$$

After $\gamma$ is inserted in the principle, the modified principle of virtual work takes the final form

$$
\begin{array}{r}
\delta W^{*}\left(\varepsilon(x), \kappa(x), \mathcal{R}_{1}(0), \mathcal{R}_{2}(0), \mathcal{M}(0), u(0), w(0), \varphi(0), u(L), w(L), \varphi(L)\right)= \\
\int_{0}^{L}\left(\left(\mathcal{N}_{\mathrm{c}}-\mathcal{N}\right) \delta \varepsilon+\left(\mathcal{M}_{\mathrm{c}}-\mathcal{M}\right) \delta \kappa\right) \mathrm{d} x- \\
{\left[u(L)-u(0)-\int_{0}^{L}\left((1+\varepsilon) \cos \varphi+\frac{\mathcal{Q}}{G A_{\mathrm{s}}} \sin \varphi\right) \mathrm{d} x+L\right] \delta \mathcal{R}_{1}(0)+} \\
{\left[w(L)-w(0)+\int_{0}^{L}\left((1+\varepsilon) \sin \varphi-\frac{\mathcal{Q}}{G A_{\mathrm{s}}} \cos \varphi\right) \mathrm{d} x\right] \delta \mathcal{R}_{2}(0)-} \\
{\left[\varphi(L)-\varphi(0)-\int_{0}^{L} \kappa \mathrm{d} x\right] \delta \mathcal{M}(0)+} \\
\left(S_{1}+\mathcal{R}_{1}(0)\right) \delta u_{1}-\left(S_{2}+\mathcal{R}_{2}(0)\right) \delta u_{2}-\left(S_{3}+\mathcal{M}(0)\right) \delta u_{3}- \\
\left(S_{4}-\mathcal{R}_{1}(L)\right) \delta u_{4}-\left(S_{5}-\mathcal{R}_{2}(L)\right) \delta u_{5}-\left(S_{6}-\mathcal{M}(L)\right) \delta u_{6}=0 .
\end{array}
$$


Note that the only unknown functions in the principle are the extensional and bending strains, $\varepsilon(x)$ and $\kappa(x)$. The remaining unknowns, displacements $u(x), w(x)$ and rotation $\varphi(x)$ as well as Lagrangian multipliers $\mathcal{R}_{1}(x), \mathcal{R}_{2}(x), \mathcal{M}(x)$ are determined from Eqns (34)-(36) and (40)-(42). This is the reason why they appear in the functional only through their boundary values.

\subsection{Finite element formulation}

The Galerkin type of the finite element numerical solution is employed. The extensional strain and the bending strain are approximated by a standard polynomial interpolation

$$
\varepsilon(x)=\sum_{n=1}^{N_{\varepsilon}} P_{n \varepsilon}(x) \varepsilon_{n}, \quad \kappa(x)=\sum_{n=1}^{N_{\kappa}} P_{n \kappa}(x) \kappa_{n},
$$

where $P_{n \varepsilon}(x)\left(n=1,2, \ldots, N_{\varepsilon}\right)$ are Lagrangian polynomials of order $N_{\varepsilon}-1$, and $P_{n \kappa}(x)$ $\left(n=1,2, \ldots, N_{\kappa}\right)$ are Lagrangian polynomials of order $N_{\kappa}-1$. The interpolation points are equidistant. $\varepsilon_{n}$ and $\kappa_{n}$ are the nodal values of extensional and bending strains, respectively. The variation of Eqn (48) gives

$$
\delta \varepsilon(x)=\sum_{n=1}^{N_{\varepsilon}} P_{n \varepsilon}(x) \delta \varepsilon_{n}, \quad \delta \kappa(x)=\sum_{n=1}^{N_{\kappa}} P_{n \kappa}(x) \delta \kappa_{n} .
$$

Inserting (48) and (49) into (47) and setting the coefficients of the independent nodal variations $\delta \varepsilon_{n}\left(n=1,2, \ldots, N_{\varepsilon}\right), \delta \kappa_{n}\left(n=1,2, \ldots, N_{\kappa}\right), \delta \mathcal{R}_{1}(0), \delta \mathcal{R}_{2}(0), \delta \mathcal{M}(0), \delta u_{i}$ $(i=1,2, \ldots, 6)$ to zero results in the system of discrete equations of the beam finite element:

$$
\begin{array}{r}
g_{n \varepsilon}=\int_{0}^{L}\left(\mathcal{N}_{\mathrm{c}}-\mathcal{N}\right) P_{n \varepsilon} \mathrm{d} x=0, \quad n=1,2, \ldots, N_{\varepsilon}, \\
g_{n \kappa}=\int_{0}^{L}\left(\mathcal{M}_{\mathrm{c}}-\mathcal{M}\right) P_{n \kappa} \mathrm{d} x=0, \quad n=N_{\varepsilon}+1, N_{\varepsilon}+2, \ldots, N_{\varepsilon}+N_{\kappa}, \\
g_{N_{\varepsilon}+N_{\kappa}+1}=u(L)-u(0)-\int_{0}^{L}\left(\left(1+\sum_{n=1}^{N_{\varepsilon}} P_{n \varepsilon} \varepsilon_{n}\right) \cos \varphi+\frac{\mathcal{Q}}{G A_{\mathrm{s}}} \sin \varphi\right) \mathrm{d} x+L=0, \\
g_{N_{\varepsilon}+N_{\kappa}+2}=w(L)-w(0)+\int_{0}^{L}\left(\left(1+\sum_{n=1}^{N_{\varepsilon}} P_{n \varepsilon} \varepsilon_{n}\right) \sin \varphi-\frac{\mathcal{Q}}{G A_{\mathrm{s}}} \cos \varphi\right) \mathrm{d} x=0, \\
g_{N_{\varepsilon}+N_{\kappa}+3}=\varphi(L)-\varphi(0)-\sum_{n=1}^{N_{\kappa}} \int_{0}^{L} P_{n \kappa}(x) \mathrm{d} x \kappa_{n}=0, \\
g_{N_{\varepsilon}+N_{\kappa}+4}=S_{1}+\mathcal{R}_{1}(0)=0,
\end{array}
$$




$$
\begin{array}{r}
g_{N_{\varepsilon}+N_{\kappa}+5}=S_{2}+\mathcal{R}_{2}(0)=0, \\
g_{N_{\varepsilon}+N_{\kappa}+6}=S_{3}+\mathcal{M}(0)=0, \\
g_{N_{\varepsilon}+N_{\kappa}+7}=S_{4}-\mathcal{R}_{1}(0)+\int_{0}^{L} p_{X} \mathrm{~d} x=0, \\
g_{N_{\varepsilon}+N_{\kappa}+8}=S_{5}-\mathcal{R}_{2}(0)+\int_{0}^{L} p_{Z} \mathrm{~d} x=0, \\
g_{N_{\varepsilon}+N_{\kappa}+9}=S_{6}-\mathcal{M}(0)-\int_{0}^{L}\left[\left(\left(1+\sum_{n=1}^{N_{\varepsilon}} P_{n \varepsilon} \varepsilon_{n}\right)-\frac{\mathcal{N}}{G A_{\mathrm{s}}}\right) \mathcal{Q}-m_{Y}\right] \mathrm{d} x=0 .
\end{array}
$$

For a given loading factor, $\lambda$, Eqns (50)-(60) constitute a system of $N_{\varepsilon}+N_{\kappa}+9$ algebraic equations $\boldsymbol{g}(\boldsymbol{x}, \lambda)=\mathbf{0}$ for $N_{\varepsilon}+N_{\kappa}+9$ unknowns, where $\boldsymbol{x}$ is the vector of unknowns. There are $N_{\varepsilon}+N_{\kappa}+3$ internal degrees of freedom: $\varepsilon_{n}\left(n=1,2, \ldots, N_{\varepsilon}\right), \kappa_{n}\left(n=1,2, \ldots, N_{\kappa}\right)$, $\mathcal{R}_{1}(0), \mathcal{R}_{2}(0), \mathcal{M}(0)$, and six external degrees of freedom: $u(0), w(0), \varphi(0), u(L), w(L)$, $\varphi(L)$ of the finite element. Unknown functions $\mathcal{R}_{1}(x), \mathcal{R}_{2}(x), \mathcal{M}(x), \varphi(x), \mathcal{N}(x), \mathcal{Q}(x)$, needed in Eqns (50)-(60), are determined from Eqns (40)-(42), (36), (11), and (12). Integrals in Eqns (36), (40) and (41) and are evaluated analytically, while the integrals in (42), (50)-(53) and (60) are evaluated numerically by Lobatto's integration (for the discussion on the choice of the numerical integration, see Planinc et al., 2001, and Saje et al., 1997).

The system of Eqns (50)-(60) is solved by Newton's method. After linearizing the equations, eliminating internal degrees of freedom, and assembling the tangent stiffness matrices of finite elements in the global coordinate system, we obtain the linear system of equations of the structure

$$
\nabla_{\boldsymbol{x}} \boldsymbol{G}\left(\boldsymbol{x}_{i}, \lambda\right) \delta \boldsymbol{x}_{i+1}=-\boldsymbol{G}\left(\boldsymbol{x}_{i}, \lambda\right), \quad \boldsymbol{x}_{i+1}=\boldsymbol{x}_{i}+\delta \boldsymbol{x}_{i+1}
$$

which is repeatedly solved for $\delta \boldsymbol{x}_{i+1}(i=0,1,2, \ldots)$ until the required accuracy is achieved. $\boldsymbol{x}_{i}$ is the vector of the external nodal unknowns at iteration $i, \boldsymbol{G}\left(\boldsymbol{x}_{i}, \lambda\right)=\boldsymbol{R}\left(\boldsymbol{x}_{i}\right)-\lambda \overline{\boldsymbol{P}}$ is the vector of the residual nodal forces, $\boldsymbol{R}$ is the vector of internal forces and $\lambda \overline{\boldsymbol{P}}$ the vector of external forces, and $\nabla_{\boldsymbol{x}} \boldsymbol{G} \equiv \boldsymbol{K}_{\mathrm{T}}$ is the Fréchet derivative of $\boldsymbol{G}$, called the structural tangent stiffness matrix.

The Crisfield arc-length method in the combination with Newton's method was used during the softening phase of the response of the reinforced concrete frame, see Crisfield (1981) and Feng et al. (1996). 


\section{Constitutive laws of concrete and reinforcing steel}

Two different constitutive laws of concrete are employed in our numerical experiments: (i) the Eurocode 2 law (1999) (henceforth referenced as the 'EC 2 model'), and (ii) the Desayi and Krishnan law (1964) (referred to as the 'DK model'). The reinforcing steel is modelled by the three-linear constitutive law.

The EC 2 stress-strain law of concrete is given by the relation

$$
\sigma_{\mathrm{c}}(D)= \begin{cases}0, & D<D_{\mathrm{cu}} \\ -f_{\mathrm{cm}} \frac{k \eta-\eta^{2}}{1+k \eta-2 \eta}, & D_{\mathrm{cu}} \leq D \leq 0 \\ 0, & D>0\end{cases}
$$

Here, $D$ is extensional strain (in $\%$ ),$\eta=\frac{D}{D_{\mathrm{c} 1}}$, and $k=-1.1 E_{\mathrm{cm}} \frac{D_{\mathrm{c} 1}}{f_{\mathrm{cm}}} ; f_{\mathrm{cm}}$ is the strength of concrete in compression (in $\mathrm{MPa}$ ); $D_{\mathrm{c} 1}$ is the strain at peak stress (in \% \%); $D_{\mathrm{cu}}$ is the ultimate strain at compression; $E_{\mathrm{cm}}$ is the secant modulus of elasticity of concrete (in $\mathrm{GPa}$ ), and $E_{\mathrm{c}, \text { nom }}$ is the nominal tangent modulus of elasticity of concrete (Fig. 2). The EC 2 model disregards the bearing capacity of concrete in tension.

(a) Eurocode 2, 1999

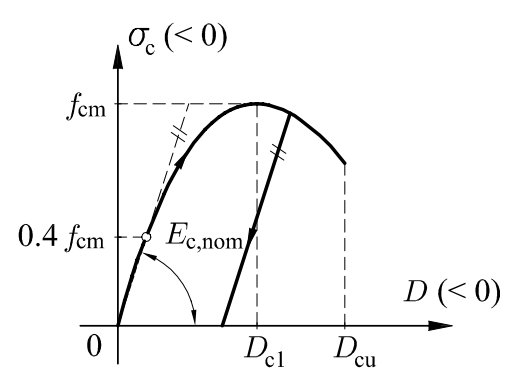

(b) Desayi and Krishnan, 1964

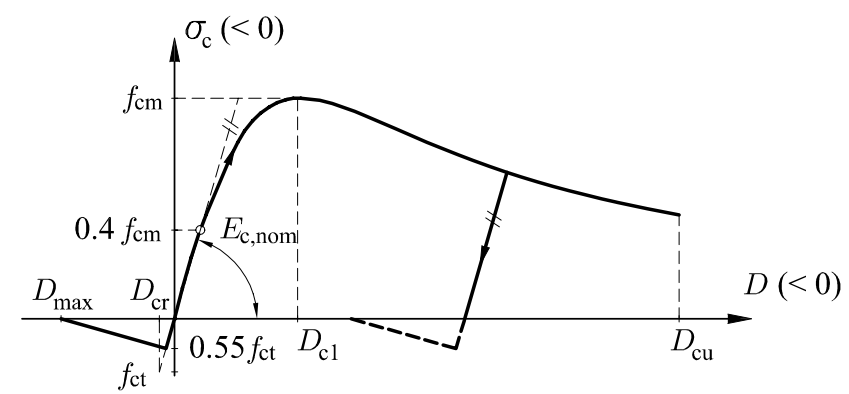

Figure 2: Constitutive laws of concrete according to (a) Eurocode 2 (1999), and (b) Desayi and Krishnan (1964).

The constitutive law of concrete according to the DK model reads

$$
\sigma_{\mathrm{c}}(D)= \begin{cases}0, & D<D_{\mathrm{cu}} \\ \frac{E_{\mathrm{c} 0} D}{1+{\frac{D}{D_{\mathrm{c} 1}}}^{2}}, & D_{\mathrm{cu}} \leq D \leq D_{\mathrm{ct} 1} \\ \frac{D_{\mathrm{ct} 2}-D}{D_{\mathrm{c} 2}-D_{\mathrm{ct} 1}} f_{\mathrm{ct}}^{\prime}, & D_{\mathrm{ct} 1} \leq D \leq D_{\mathrm{ct} 2} \\ 0, & D>D_{\max }\end{cases}
$$


Here $D_{\mathrm{ct} 1}=0.55 D_{\mathrm{cr}} ; D_{\mathrm{ct} 2}=D_{\max } ;$ and $E_{\mathrm{c} 0}=-\frac{2 f_{\mathrm{cm}}}{D_{\mathrm{ct} 1}}$ is the initial tangent elastic modulus of concrete. Although the DK model accounts for the bearing capacity of concrete in tension ('tension stiffening'), we assume that the tension part of the constitutive law follows the proposal by Bergan and Holand (1979). We take $f_{\mathrm{ct}}^{\prime} \approx 0.55 f_{\mathrm{ct}}\left(f_{\mathrm{ct}}\right.$ is the strength of concrete in tension) and $D_{\text {ct2 }} \approx 0.7 \%$ (Fig. 2). Note that the ultimate strain at compression, $D_{\text {cu }}$, depends considerably on stirrups (Desayi and Krishnan, 1964).

The two constitutive models are jointly displayed in Fig. 2. Observe that both models take into account the softening of concrete in compression. The unloading is considered to be elastic in both models (Fig. 2). An initial yield stress at compression is $0.4 f_{\mathrm{cm}}$.

The behaviour of reinforcing streel in tension and compression is modelled by the threelinear law:

$$
\sigma_{\mathrm{s}}(D)=\left\{\begin{array}{ll}
E_{\mathrm{s}} D, & |D| \leq D_{\mathrm{y} 1} \\
\left(f_{\mathrm{y}}+E_{\mathrm{p}}\left(|D|-D_{\mathrm{y} 1}\right)\right) \operatorname{sgn}(D), & D_{\mathrm{y} 1}<|D| \leq D_{\mathrm{y} 2} \\
\left(f_{\mathrm{y}}+E_{\mathrm{p}}\left(D_{\mathrm{y} 2}-D_{\mathrm{y} 1}\right)\right)\left(1-\frac{|D|-D_{\mathrm{y} 2}}{D_{\mathrm{yu}}-D_{\mathrm{y} 2}}\right) \operatorname{sgn}(D), & D_{\mathrm{y} 2}<|D| \leq D_{\mathrm{yu}} \\
0, & |D|>D_{\mathrm{yu}}
\end{array} .\right.
$$

$E_{\mathrm{s}}$ is the elastic modulus of streel; $E_{\mathrm{p}}$ is its hardening modulus in the plastic region; $f_{\mathrm{y}}$ is the yield stress; $D_{\mathrm{y} 1}$ is the related strain; $D_{\mathrm{y} 2}$ is the strain at the peak stress; and $D_{\mathrm{yu}}$ is the ultimate strain in steel (Fig. 3). The isotropic-type of hardening is assumed.

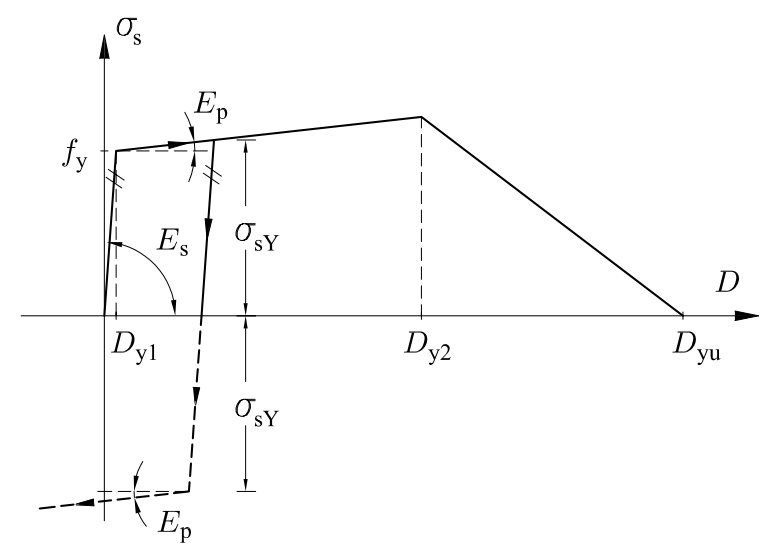

Figure 3: Constitutive law of reinforcing steel.

Constitutive models are crucial in the computation of the cross-sectional constitutive forces $\left(\mathcal{N}_{\mathrm{c}}\right.$ and $\mathcal{M}_{\mathrm{c}}$, see Eqns (15) and (17)), and the components of the tangent constitutive matrix of the cross-section. These quantities are obtained by the integration over the 
cross-section, which consists of concrete and steel. For the integration over concrete parts of the cross-section, the individual part is divided into $l_{\max }$ trapezoidal layers (Fig. 4). The contribution of each layer is obtained by the Gaussian integration. The contribution of the reinforcement is taken point-wise. We assume a complete extensional strain conformity between concrete and steel. The constitutive axial force at the cross-section is obtained from the relation

$$
\mathcal{N}_{\mathrm{c}}=\mathcal{N}_{\mathrm{c}, \mathrm{c}}+\mathcal{N}_{\mathrm{c}, \mathrm{s}}=\sum_{l=1}^{l_{\max }} b_{l} \int_{\Delta z_{l}} \sigma_{\mathrm{c}} \mathrm{d} z+\sum_{k=1}^{k_{\max }} \sigma_{\mathrm{s}}\left(D_{\mathrm{s}}^{k}\right) A_{\mathrm{s}}^{k} .
$$

Here, as previously, index "c" denotes the contribution of concrete, and index "s" that of steel. Likewise, the constitutive moment is determined from the relation

$$
\mathcal{M}_{\mathrm{c}}=\mathcal{M}_{\mathrm{c}, \mathrm{c}}+\mathcal{M}_{\mathrm{c}, \mathrm{s}}=\sum_{l=1}^{l_{\max }} b_{l} \int_{\Delta z_{l}} \sigma_{\mathrm{c}} z \mathrm{~d} z+\sum_{k=1}^{k_{\max }} \sigma_{\mathrm{s}}\left(D_{\mathrm{s}}^{k}\right) z_{\mathrm{s}}^{k} A_{\mathrm{s}}^{k}
$$

$A_{\mathrm{S}}^{k}$ is the cross-sectional area of the $k^{\text {th }}$ reinforcing bar, and $z_{\mathrm{S}}^{k}$ is the $z$-coordinate of its centroid.

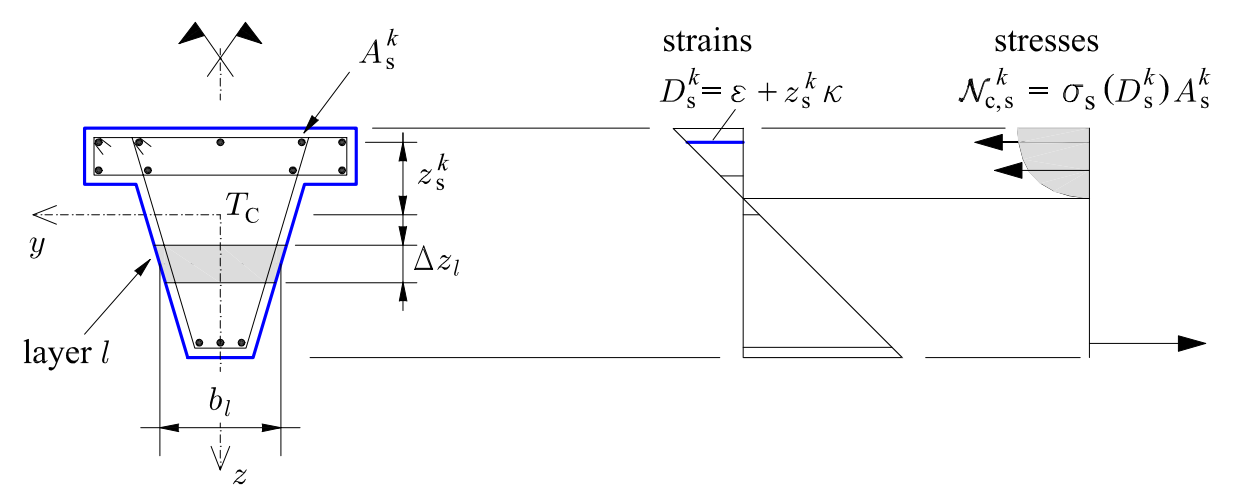

Figure 4: Typical cross-section.

\section{Numerical examples}

We show three numerical examples and make comparisons of the results with the tests reported in literature (Espion, 1993; Ferguson and Breen, 1966; Cranston, 1965).

The polynomials with equal degree are chosen for the interpolation of the extensional and bending strains, i.e. $N=N_{\varepsilon}=N_{\kappa}$. A finite element with $N^{\text {th }}$ degree polynomial, and $M$-point numerical integration along the axis of an element, is denoted by $E_{N-M}$. 
Lobatto's integration is employed for the integration with respect to $x$, and Gaussian integration over the cross-sections.

\subsection{Foure's column}

Our first example is Foure's column (see Fig. 5). This reinforced concrete column was chosen by the RILEM Technical Committee TC 114 as one of the bench-mark problems for testing the computational models and computer programmes for reinforced concrete structures. The column was subjected to an eccentric, slowly increasing axial force until the collapse took place. The results of the laboratory test were documented by Espion (1993). The geometric, material, and loading data of the column are given in Fig. 5.
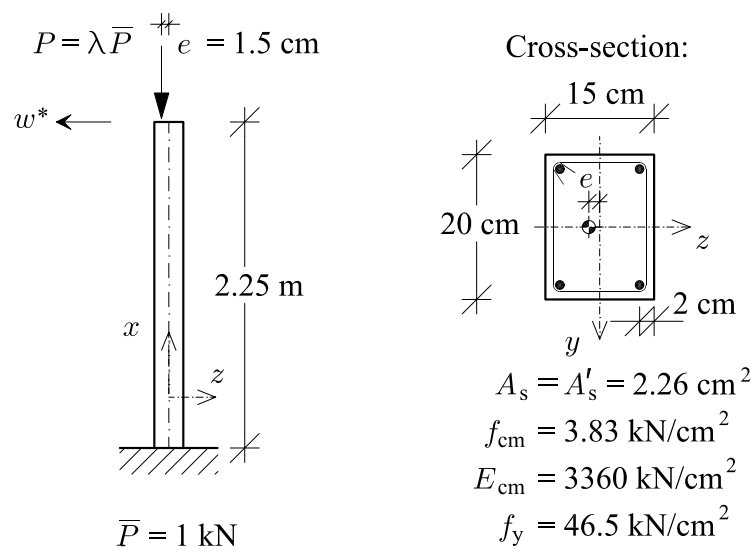

Figure 5: Foure's column. Geometric, material, and loading data.

Only three material parameters are given by Espion (1993): the compressive strength of concrete, $f_{\mathrm{cm}}$, elastic modulus of concrete, $E_{\mathrm{cm}}$, and strength of steel, $f_{\mathrm{y}}$. The remaining material parameters needed for our analysis are estimated on the basis of given strength and EC 2. They are: the peak and ultimate compression strains of concrete, $D_{\mathrm{c} 1}=$ $-2.3 \%$ and $D_{\mathrm{cu}}=-3.5 \%$; the elastic modulus of steel, $E_{\mathrm{s}}=20000 \mathrm{kN} / \mathrm{cm}^{2}$; the hardening modulus of steel, $E_{\mathrm{p}}=0 \mathrm{kN} / \mathrm{cm}^{2}$ (no strain-hardening), and its ultimate strains $D_{\mathrm{y} 2}=D_{\mathrm{yu}}=20 \%$ (no strain-softening). The EC 2 material model of concrete is employed.

The measured ultimate critical load was $P_{\mathrm{cr}}^{\text {test }}=454 \mathrm{kN}$, and the related free-end lateral deflection $w_{\text {cr,test }}^{*}=2.61 \mathrm{~cm}$ (Espion, 1993). The collapse of the column took place at load $P_{\text {col }}^{\text {test }}=445 \mathrm{kN}$ and deflection $w_{\text {col,test }}^{*}=3.21 \mathrm{~cm}$. The graphs in Fig. 6 show the 
comparisons between the test and the calculated values for the free-end lateral deflection, $w^{*}$, as a function of load $P$. As clearly observed, the present result and the result of the test agree well. The load-deflection curve was obtained by Crisfield's arc-length method with the initial arc-length $\Delta s=0.25$. The results shown in Fig. 6 were obtained by the use of only two $E_{4-5}$ finite elements. Such a coarse mesh is sufficient, as will be shown later. Five layers were used for the integration over the concrete cross-section, and 10-point Gaussian integration within each of the layers. The ultimate critical load is characterized by the zero determinant of the tangent stiffness matrix of the structure. The bisection was employed to determine the root of the determinant.

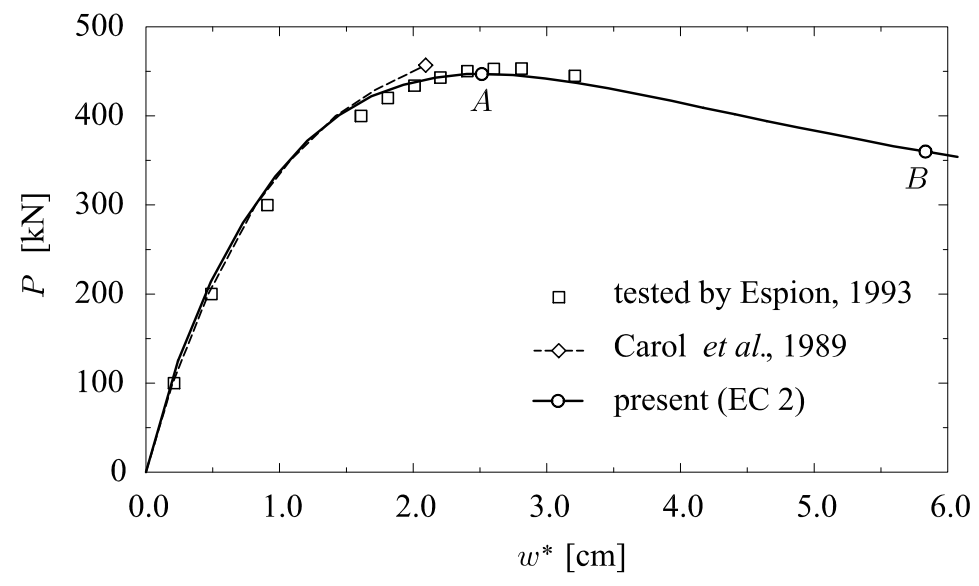

Figure 6. Foure's column. Loading factor vs lateral deflection curve.

The comparison between the test and the numerical results.

The calculated ultimate critical load, $P_{\text {cr }}^{\text {calc }}=447 \mathrm{kN}$, is only $7 \mathrm{kN}$ smaller than the measured one (see point $A$ in Fig. 6). The related calculated tip deflection, $w_{\mathrm{cr}, \text { calc }}^{*}=$ $2.51 \mathrm{~cm}$, differs from the measured one by only $0.1 \mathrm{~cm}$. The corresponding maximum compression strain in concrete at the clamped end of the column is $D_{\mathrm{c}}=-1.32 \%$ and the related longitudinal stress is $\sigma_{\mathrm{c}}=-3.18 \mathrm{kN} / \mathrm{cm}^{2}$; the maximum strain and stress in steel are also compressive $\left(D_{\mathrm{s}}=-1.08 \%\right.$ and $\left.\sigma_{\mathrm{s}}=-21.61 \mathrm{kN} / \mathrm{cm}^{2}\right)$. Once the ultimate load is reached, the load-deflection curve starts decreasing. The calculation shows, that at $P_{B}=360 \mathrm{kN}$ and $w_{B}^{*}=5.83 \mathrm{~cm}$ ( $B$ marks the point in the load-deflection curve in Fig. 6) the maximum compressive strain in concrete amounts to $D_{\mathrm{c}}=-2.26 \%$, while the related stress is $\sigma_{\mathrm{c}}=-3.82 \mathrm{kN} / \mathrm{cm}^{2}$. The fact that $\left|D_{\mathrm{c}}\right|<D_{\mathrm{c} 1}=2.3 \%$ indicates that concrete is still in the hardening regime. The most strained steel bar at the clamped 
end is now in tension, $D_{\mathrm{s}}=1.86 \%\left(\sigma_{\mathrm{s}}=37.21 \mathrm{kN} / \mathrm{cm}^{2}\right)$. As the yield strain of steel is $D_{\mathrm{y} 1}=f_{\mathrm{y}} / E_{\mathrm{s}}=2.325 \%>1.86 \%$, it is clear that steel has behaved elastically up to this moment.

Shortly after point $B$ in Fig. 6 is reached, at $P=297 \mathrm{kN}, w^{*}=7.71 \mathrm{~cm}$, the tangent stiffness matrix of the column becomes singular again. Unfortunatelly, this critical point does not coincide with the physical collapse of the column measured in the experiment it took place a lot earlier, see Fig. 6 (Espion, 1993).

The calculations that use the DK model and employ the same material parameters as EC 2, give virtually identical results compared to the EC 2 model. Therefore, for the present problem, the two models are equivalent. Fig. 6 also shows the load-deflection curve of Carol and Muricia (1989), who used Sargin's model of concrete and the bi-linear elastic-plastic model for the reinforcement. They employed the 2nd order beam theory to capture geometrical non-linearity. Their estimate of the critical load is fairly good, whereas their estimate of the critical deflection is not.

It is instructive to study the effect of the number of elements, the degree of interpolation, and the order of Lobatto's integration on the accuracy of the critical load. The results are presented in Fig. 7. They are compared to the highly accurate solution obtained by employing 16 elements $E_{8-9}$, and marked by $P_{\mathrm{cr}, 16}$. Fig. 7 a shows the effect of the number of elements. We see that the increase in the number of elements is followed by a substantial decrease in the error. Fig. 7b shows the effect of the integration order when using one-element mesh to model the column. This time the increase in the integration order does not unconditionally mean the decrease in the error (see Planinc et al., 2001, for the discussion). Please notice that one-element solution is already very accurate: for one element $E_{3-4}$, the relative error is $\Delta P_{\mathrm{cr}}=0.11 \%$; for one element $E_{4-5}$, it is only $0.009 \%$.

\subsection{Square frame}

Our second example is a square frame, tested by Ferguson and Breen (1966) as frame $L_{3}$. The results of the test were presented by Gunnin et al. (1977). The geometry of crosssections of columns and beams of the frame along with material data for concrete and steel $\left(f_{\mathrm{cm}}, f_{\mathrm{y}}, E_{\mathrm{s}}\right)$ as offered by Gunnin et al. (1977) are displayed in Fig. 8. The remaining material parameters needed for our analysis but not stated in Gunnin et al. (1977) are 
(a)

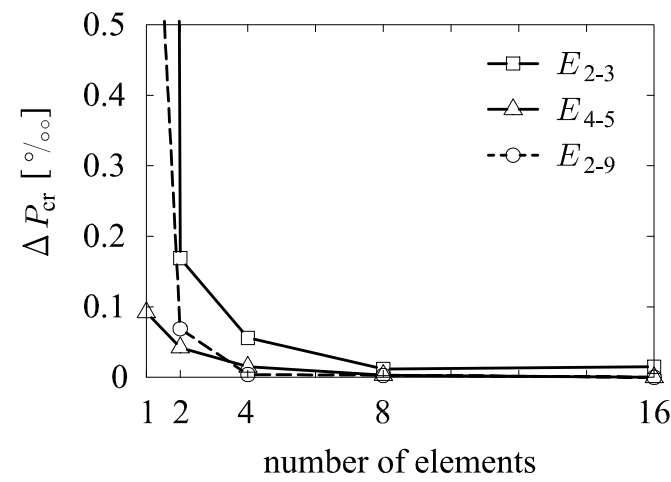

(b) one element

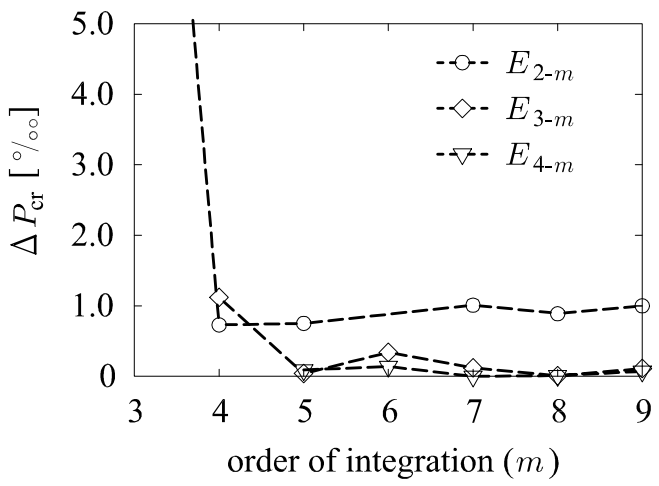

Figure 7. Foure's column. The error of $P_{\text {cr }}$ vs (a) number of finite elements, (b) order of numerical integration; $\Delta P_{\mathrm{cr}}=\left|\frac{P_{\mathrm{cr}}-P_{\mathrm{cr}, 16}}{P_{\mathrm{cr}, 16}}\right|$.

estimated on the basis of the given strengths of concrete and steel, and EC 2. They are: elastic modulus of concrete, $E_{\mathrm{cm}}=2800 \mathrm{kN} / \mathrm{cm}^{2}$; peak and ultimate compression strains of concrete, $D_{\mathrm{c} 1}=-1.85 \%$ and $D_{\mathrm{cu}}=-3.5 \%$; hardening modulus of steel, $E_{\mathrm{p}}=0 \mathrm{kN} / \mathrm{cm}^{2}$ (no strain-hardening), and its ultimate strains $D_{\mathrm{y} 2}=D_{\mathrm{yu}}=20 \%$ (no strain-softening). The frame was tested in the laboratory under slowly increasing vertical and horizontal forces until the frame collapsed.
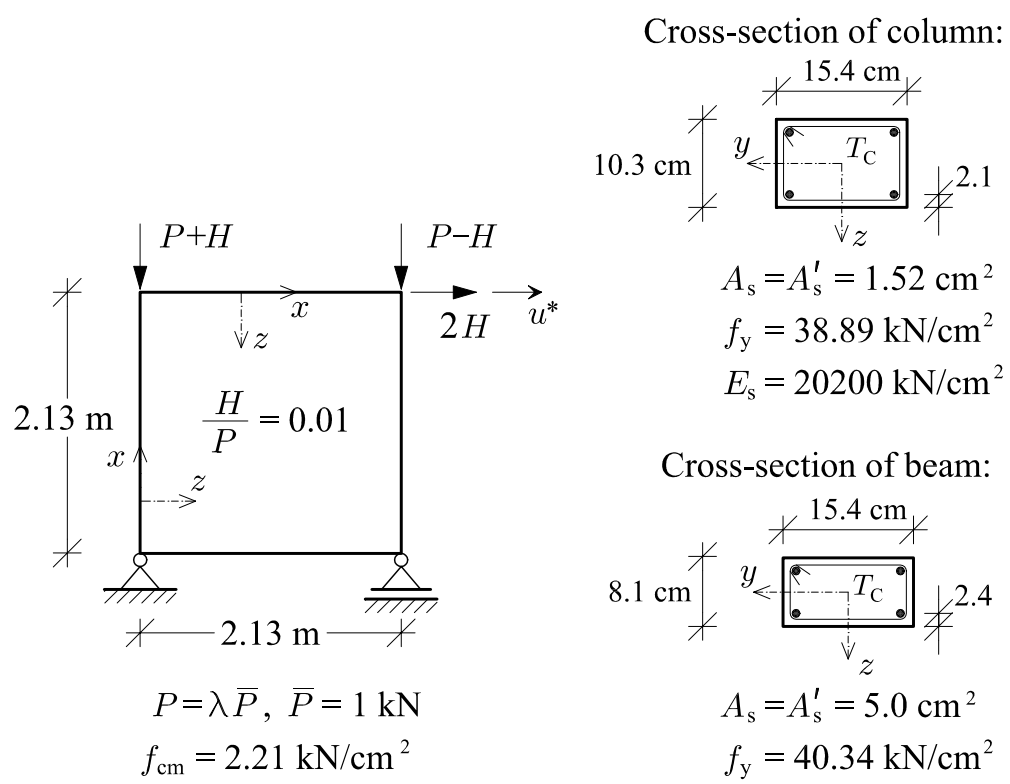

$$
\begin{aligned}
& A_{\mathrm{s}}=A_{\mathrm{s}}^{\prime}=1.52 \mathrm{~cm}^{2} \\
& f_{\mathrm{y}}=38.89 \mathrm{kN} / \mathrm{cm}^{2} \\
& E_{\mathrm{s}}=20200 \mathrm{kN} / \mathrm{cm}^{2}
\end{aligned}
$$

Cross-section of beam:

$$
\begin{aligned}
8.1 \mathrm{~cm}{ }_{*} & { }^{+15.4 \mathrm{~cm}} \\
A_{\mathrm{s}} & =A_{\mathrm{s}}^{\prime}=5.0 \mathrm{~cm}^{2} \\
f_{\mathrm{y}} & =40.34 \mathrm{kN} / \mathrm{cm}^{2} \\
E_{\mathrm{s}} & =20200 \mathrm{kN} / \mathrm{cm}^{2}
\end{aligned}
$$

Figure 8. Square frame of Ferguson and Breen (1966). Geometric, material, and loading data. 
The load-horizontal displacement curve was determined by Crisfield's arc-length method. The initial arc-length was $\Delta s=0.75$. The results in Fig. 9 were obtained by the mesh consisting of four $E_{4-5}$ finite elements, i.e., each column or beam was modelled by one element. Five layers were used for the integration over the concrete cross-section, and 10-point Gaussian integration within each of them. The EC 2 model was employed along with the no-tension assumption of concrete.

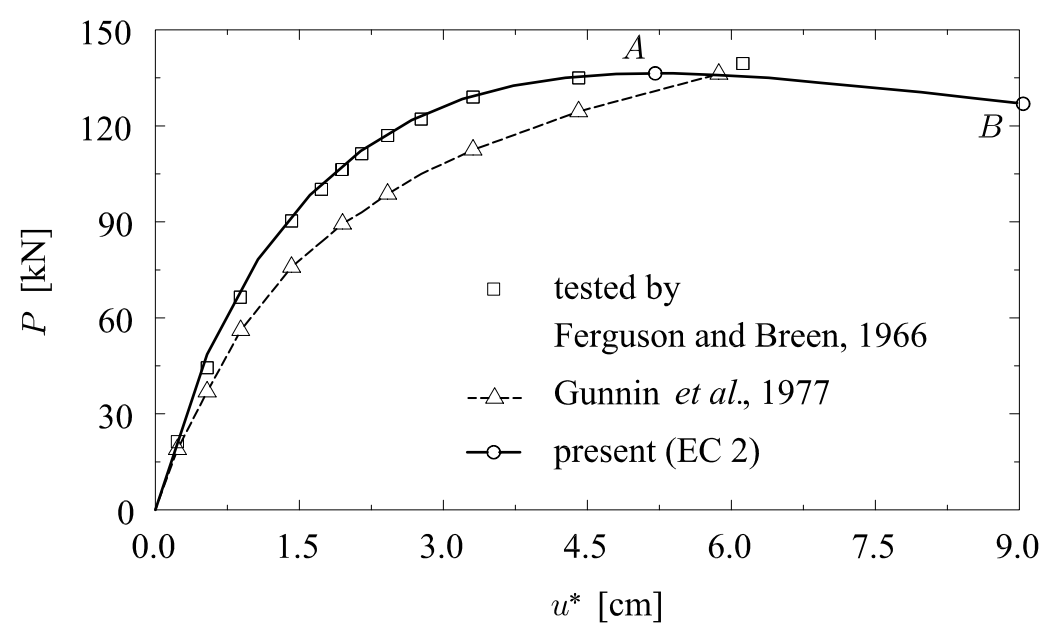

Figure 9. Square frame. Load vs horizontal deflection curve.

The ultimate critical load measured in the test was $P_{\mathrm{cr}}^{\mathrm{test}}=141 \mathrm{kN}$, and the corresponding horizontal displacement was $u_{\mathrm{cr}, \text { test }}^{*}=6.11 \mathrm{~cm}$. The calculated critical load (point $A$ in Fig. 9) agrees well with the measured one, and is $P_{\mathrm{cr}}^{\text {calc }}=136.4 \mathrm{kN}$. The related horizontal displacement of the point of application of force $2 H$ is $u_{\mathrm{cr}, \text { calc }}^{*}=5.21 \mathrm{~cm}$. Fig. 9 shows the comparisons for the horizontal displacement, $u^{*}$, as a function of load $P$. As we can see, the results agree nicely. The numerical solution of Gunnin et al. (1977) is also displayed. Their solution is rather imprecise, which may be due to an insufficiently accurate modelling of the geometric non-linearity by the ' $P-\Delta$ method'.

An equal maximum compression strain in concrete at the ultimate critical load appears in two cross-sections, both in the right end of the beams: in the lower beam, at its upper side, and in the upper beam, at its lower side of the cross-section. The maximum compression strain is $D_{\mathrm{c}}=-1.73 \%$. The extensional strain in steel reinforcing bars is also maximal at these cross-sections $\left(D_{\mathrm{s}}=1.25 \%\right.$ \% $)$.

Once the ultimate critical load is reached, the load-horizontal displacement curve starts 
decreasing. At the load $P_{B}=126.8 \mathrm{kN}$ and the horizontal displacement $u_{B}^{*}=9.04 \mathrm{~cm}$ (marked by point $B$ on the load-displacement curve in Fig. 9), the maximum compressive strain in concrete becomes as high as $D_{\mathrm{c}}=-3.01 \%$, which indicates the softening of concrete; by contrast, the most strained steel bar which remains in tension $\left(D_{\mathrm{s}}=1.89 \%\right.$ \% behaves elastically.

A detailed study of errors in the horizontal displacement, $u^{*}$, and the constitutive moment at the tip of the right-hand column, $\mathcal{M}_{\mathrm{c}}^{*}$, is now made as a function of the type and number of finite elements. The errors at load $P=130 \mathrm{kN}$, which is roughly $95 \%$ of the ultimate critical load, are presented. The comparisons are shown in Figs. 10a and 10b, where various results are compared to those obtained with 32 elements $E_{8-9}$, i.e. 8 very accurate elements per column or beam. These results are denoted by $u_{32}^{*}$ and $\mathcal{M}_{\mathrm{c}, 32}^{*}$. As observed from the graphs, the relative errors are small even if only four elements $E_{4-5}$ are used; e.g. the relative error in the horizontal displacement is about $1 \%$, and the error in the constitutive moment is about $0.5 \%$. Note that the order of numerical integration greater than $N+1$ somewhat improves the results for displacements, but makes the results for constitutive moments substantially worse (see Fig. 10b); e.g. employing four elements $E_{4-5}$ makes the error in $\Delta \mathcal{M}_{\mathrm{c}}^{*}$ to be $0.57 \%$ in contrast to $2.33 \%$ when four elements $E_{4-9}$ are used. This interesting result is in agreement with the discussion by Planinc et al. (2001) for elastic-plastic material.

(a)

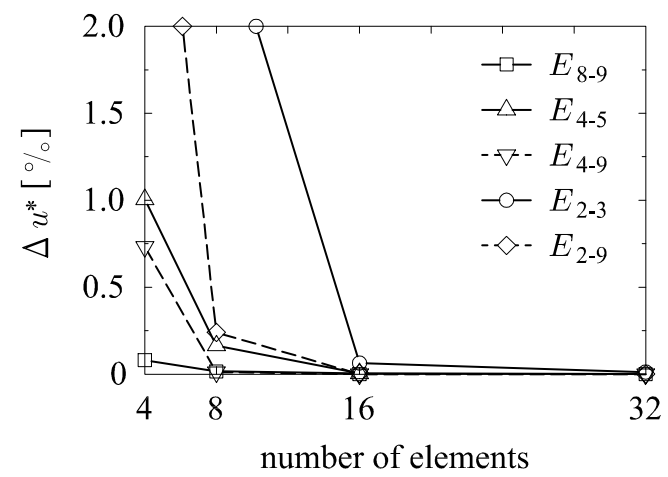

(b)

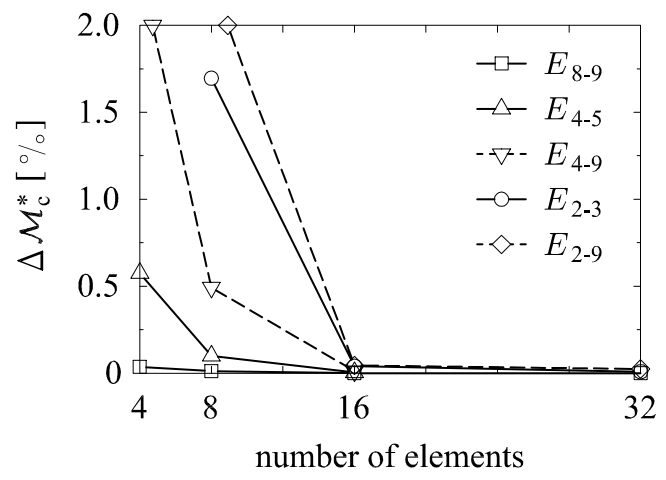

Figure 10. Square frame. The accuracy of results as a function of number and type of finite elements at load $P=130 \mathrm{kN}$. (a) Horizontal displacement, $\Delta u^{*}=\left|\frac{u^{*}-u_{32}^{*}}{u_{32}^{*}}\right|$; (b) constitutive moment at the top of the right-hand column, $\Delta \mathcal{M}_{\mathrm{c}}^{*}=\left|\frac{\mathcal{M}_{\mathrm{c}}^{*}-\mathcal{M}_{\mathrm{c}, 32}^{*}}{\mathcal{M}_{\mathrm{c}, 32}^{*}}\right|$. 
In design the accuracy of stresses is of great importance. The stresses are determined from the axial and shear forces and the bending moment. In numerical solutions, there are two kinds of forces and moments, i.e. the equilibrium and the constitutive ones (see Section 2). The smaller the difference between the two types of forces, the more accurate the solution; hence, the differences $\left|\mathcal{N}_{\mathrm{c}}-\mathcal{N}\right|$ and $\left|\mathcal{M}_{\mathrm{c}}-\mathcal{M}\right|$ are the indicators of the accuracy of the solution. Fig. 11 shows the graphs of $\left|\mathcal{M}_{\mathrm{c}}-\mathcal{M}\right|$ for the right-hand column of the frame at $P=130 \mathrm{kN}$ for cases where one, two, three, and four finite elements per column or beam are applied. The results of two different-order elements, $E_{4-5}$ and $E_{4-9}$, are displayed. The positions of the Lobatto integration points are shown by circles (o). For element $E_{4-5}$, where the number of interpolation points coincides with the order of Lobatto's integration, the two moments coincide at the integration points. Otherwise this is not the case (see the results of element $E_{4-9}$ ); however, the peak and the overall errors now appear to be much smaller. Observe that the error diminishes exponentially with the growth of the number of elements (see Fig. 11a-d).

The results show that very reliable values for the bending moment are obtained at integration points if the number of interpolation points coincides with the order of Lobatto's integration. For example, the largest bending moment at the top of the right-hand column is $\mathcal{M}_{\max }=356.09 \mathrm{kNcm}$ and $358.15 \mathrm{kNcm}$ for one and four-element meshes, respectively, the difference being only about $0.6 \%$. Similar conclusions are valid for the axial forces. Note that substantially larger errors would be obtained with classical finite elements if the consistency conditions (18)-(20) were not applied.

\subsection{Cranston's portal frame}

A two-hinge pinned reinforced concrete frame, tested by Cranston (1965) as frame $P_{2}$, and analysed by Lazaro and Richards (1973) and Bažant et al. (1987a), is studied in this section. The behaviour of Cranston's frame $P_{2}$ is characterized by a massive strainsoftening of concrete, which triggers localizations of deformations and an overall softening of the structure. A special approach is needed to model the strain-softening of material numerically. In this paper we assume that the localization of deformation takes place in a small, yet a finite-length region of the beam, $\Delta L_{\mathrm{m}}$, and determine its length from the

fracture energy of concrete in compression, $G_{f}^{\mathrm{c}}$, as described in Coleman and Spacone (2001). This approach is often called the local continuum approach. 
(a) one element

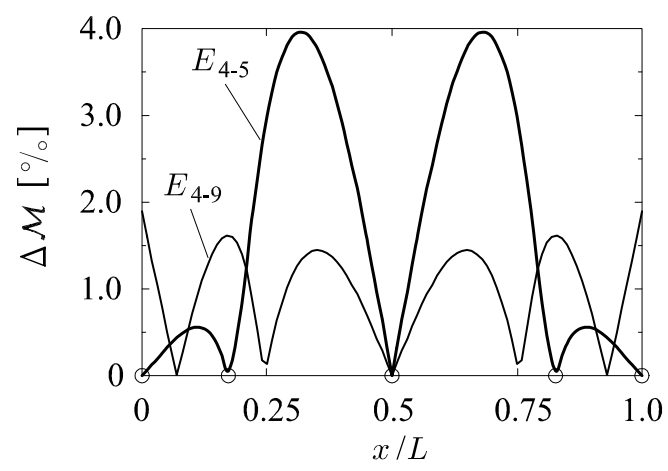

(c) 3 elements

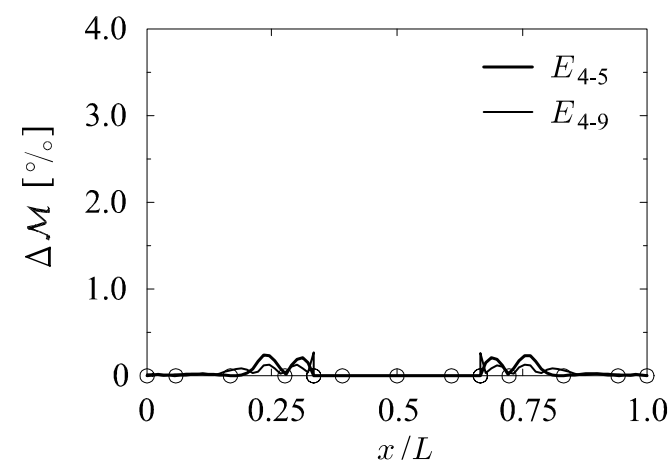

(b) two elements

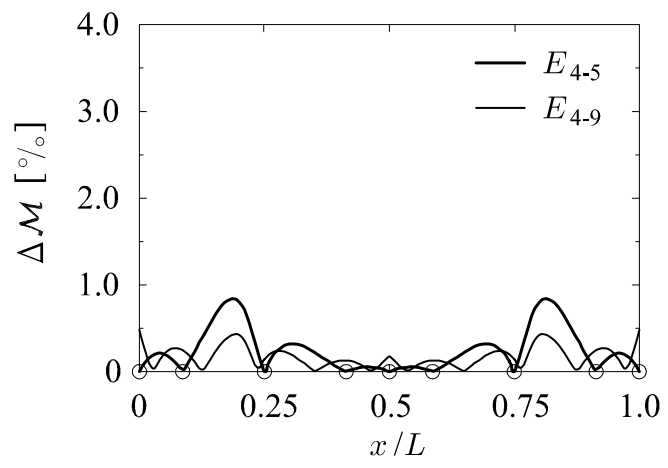

(d) 4 elements

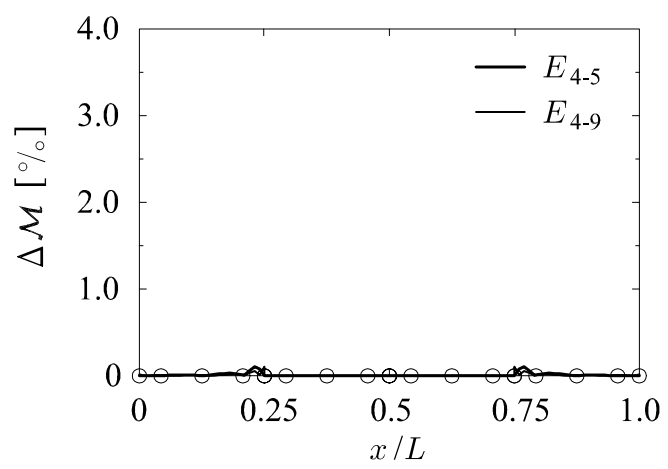

Figure 11. Square frame. The graph of $\Delta \mathcal{M}=\left|\frac{\mathcal{M}_{\mathrm{c}}-\mathcal{M}}{\mathcal{M}_{\max }}\right|$ vs $x / L$ for the right-hand column.

$$
P=130 \mathrm{kN} \text {. }
$$

The descriptive data are displayed in Fig. 12. Only two material parameters are given in Cranston (1965), i.e. $f_{\mathrm{cm}}$ and $f_{\mathrm{y}}$. For the remaining parameters, we made estimates using the given strengths and EC $2: E_{\mathrm{cm}}=3150 \mathrm{kN} / \mathrm{cm}^{2} ; D_{\mathrm{c} 1}=-2.3 \% \circ E_{\mathrm{s}}=20000 \mathrm{kN} / \mathrm{cm}^{2}$; $E_{\mathrm{p}}=200 \mathrm{kN} / \mathrm{cm}^{2} ; D_{\mathrm{y} 2}=10 \%$ and $D_{\mathrm{yu}}=300 \%$. The same parameters were taken for the DK model. The DK model needs two additional parameters to consider the tensionstiffening, i.e. $D_{\text {ct1 }}=D_{\text {cr }}^{\prime}=0.055 \%$ and $D_{\max }=0.7 \%$. Similar, but not equal parameters were assumed by Lazaro and Richards (1973) and Bažant et al. (1987a). We assumed that the fracture energy of concrete in compression is $G_{f}^{\mathrm{c}}=20 \mathrm{~N} / \mathrm{mm}$ (Jansen and Shah, 1997), and the ultimate fracture strain in compression $D_{\mathrm{cu}}=-50 \%$. As a results, the length of the localization zone of concrete is $\Delta L_{\mathrm{m}}=4 \mathrm{~cm}$ (Coleman and Spacone, 2001).

The initial arc-length in Crisfield's arc-length method was $\Delta s=0.5$. The results in Fig. 13 were obtained by the use of 8 finite elements $E_{4-5}$ and 13 short constant-strain 
elements $E_{0-1}$ of length $\Delta L_{\mathrm{m}}=4 \mathrm{~cm}$. The finite-element mesh was designed in such a way that short elements could capture the localization of deformations. Thus, the beam was modelled by (in the following order from left to right): one short element $E_{0-1}$ at the left node of the beam (length: $4 \mathrm{~cm}$ ), two elements $E_{4-5}$ (lengths: $51 \mathrm{~cm}$ and $55 \mathrm{~cm}$ ), 11 short elements between the applied forces, two elements $E_{4-5}$ and one short element. The columns were divided into two elements $E_{4-5}$ each. The finite element mesh is depicted in Fig. 12a. Ten layers were used for the integration over the concrete cross-sections, and the 10-point Gaussian integration was employed within each of them. For the sake of comparison with other numerical studies (Bažant et al., 1987a, and Lazaro and Richards, 1973), only one half of the frame was analysed assuming its symmetry.

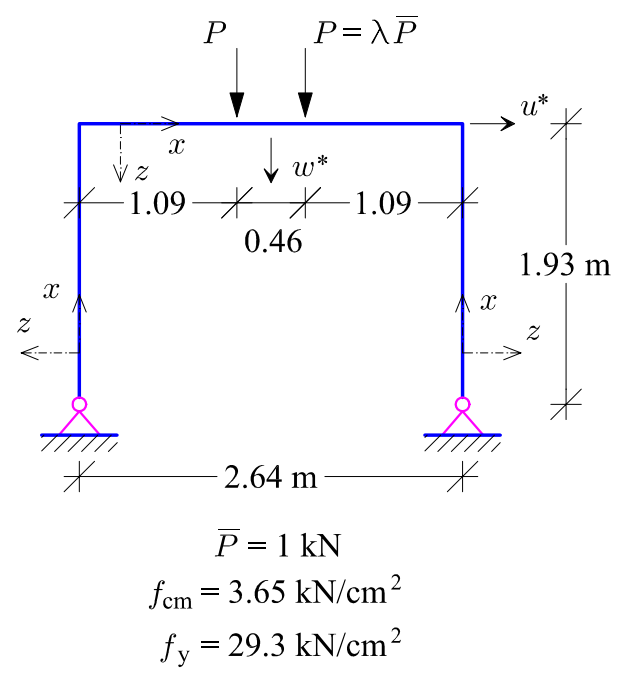

(a) Case 1

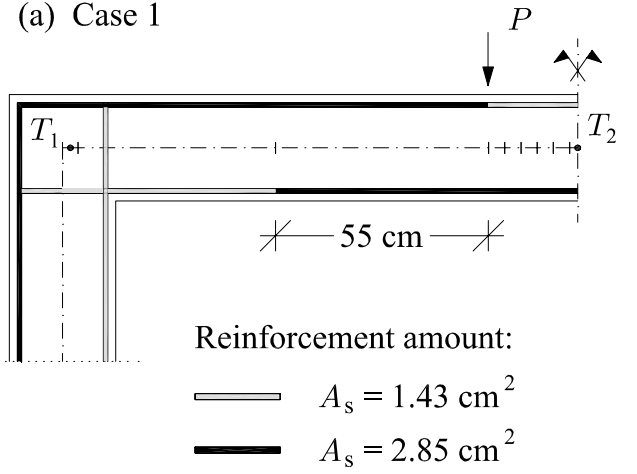

$$
\begin{array}{cc}
\text { Cross-section } & \text { Cross-section } \\
\text { at } T_{1}, T_{3}, T_{4}: & \text { at } T_{2}, T_{5}:
\end{array}
$$

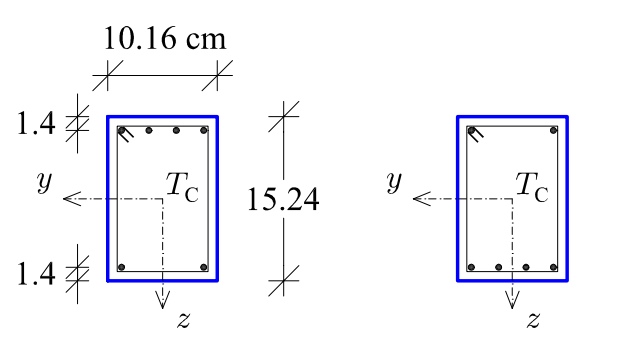

$$
\begin{array}{ll}
A_{\mathrm{s}}=1.43 \mathrm{~cm}^{2} & A_{\mathrm{s}}=2.85 \mathrm{~cm}^{2} \\
A_{\mathrm{s}}^{\prime}=2.85 \mathrm{~cm}^{2} & A_{\mathrm{s}}^{\prime}=1.43 \mathrm{~cm}^{2}
\end{array}
$$

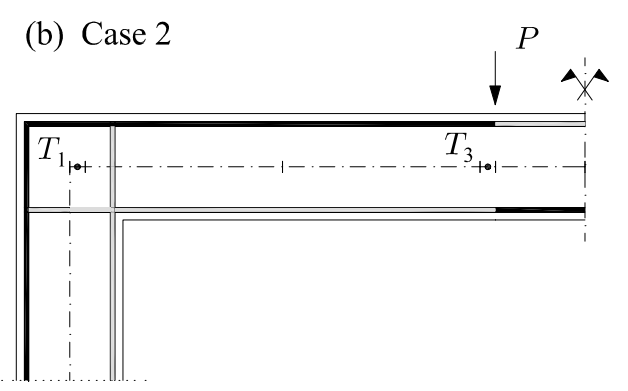

Figure 12. Cranston's portal frame. Geometrical, material, and loading data. (a) Original position of reinforcement (Case 1); (b) alternative position of reinforcement (Case 2). 


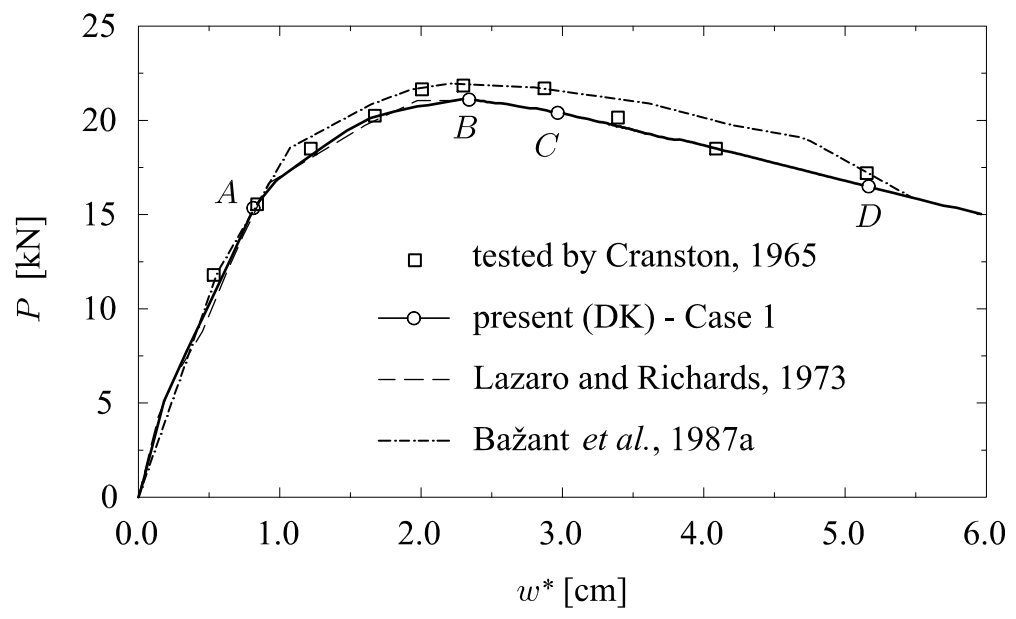

Figure 13. Cranston's portal frame. Load-mid-point deflection curves.

Fig. 13 shows graphs of the vertical deflection, $w^{*}$, at mid-point $T_{2}$ as a function of the load, $P$. The results are compared to those obtained by Cranston (1965). A good overall agreement between the two results may be observed. The numerical results by Bažant et al. (1987a) and Lazaro and Richards (1973) are also displayed (note that their solutions employed the geometrically linear theory).

The form of the load-deflection curve is roughly three-linear. Up to point $A$, the frame behaves virtually elastically. From $A$ to $B$, some of the cross-sections have partly plastified, which results in a decreased stiffness of the frame. At $B$ the determinant of the tangent stiffness matrix of the frame becomes zero $\left(\operatorname{det} \boldsymbol{K}_{\mathrm{T}}=0\right)$. The analysis of the matrix eigenvectors shows that the limit load of the frame and not its bifurcation point is reached. Simultaneously, the determinant of the tangent constitutive matrix of the beam cross-section at point $T_{1}$ (and also at point $T_{1}^{\prime}$ ) becomes zero $(\operatorname{det} \boldsymbol{C}=0$, see Fig. $14 \mathrm{~b}$ ), which indicates that the ultimate bearing capacity of both, the cross-section and the frame, is reached. In the subsequent deformation, the frame exhibits a softening behaviour, both globally and locally at point $T_{1}$. At point $C$ of the load-displacement curve, the ultimate bearing capacity is simultaneously reached at all cross-sections between the forces (point $T_{2}$ will represent these points), see the curve marked by $\operatorname{det} \boldsymbol{C}$ in Fig. 14d. These crosssections soften afterwards.

As already stated, at the ultimate critical load, $P_{\mathrm{cr}}=21.10 \mathrm{kN}$, both, the determinant of the tangent stiffness matrix of the frame, and the determinant of the tangent constitutive matrix of the beam cross-section at $T_{1}$ become zero. The related largest compression strain 
in concrete at $T_{1}$ is $D_{\mathrm{c}}=-2.44 \%$ o which indicates that some of the fibres in concrete have entered the softening regime before the $\operatorname{det} \boldsymbol{C}$ becomes zero. The same holds for the steel bars, where the tensile strain $D_{\mathrm{s}}=11.32 \%$ is greater than $D_{\mathrm{y} 2}=10 \%$, where the steel starts softening. The related bending moment is $\mathcal{M}=-1165 \mathrm{kNcm}$, which is only a little less than the value obtained by Cranston (1965) on the basis of the measured strain distributions $\left(\mathcal{M}_{\text {Cranston }}=-1247 \mathrm{kNcm}\right)$ (see Fig. 15a). These quantities at the mid-point cross-section, point $T_{2}$, are: the maximal compression strain in concrete is $D_{\mathrm{c}}=-1.96 \%$ o the tension strain in steel is $D_{\mathrm{s}}=7.75 \%$, and the bending moment is $\mathcal{M}=1147 \mathrm{kNcm}$ (Fig. 15b). Figures 14c-d show that the cross-section is still in the hardening regime. The deflection at the mid-point $T_{2}$ is $w_{\mathrm{cr}}^{*}=2.34 \mathrm{~cm}$.

(a) point $T_{1}$

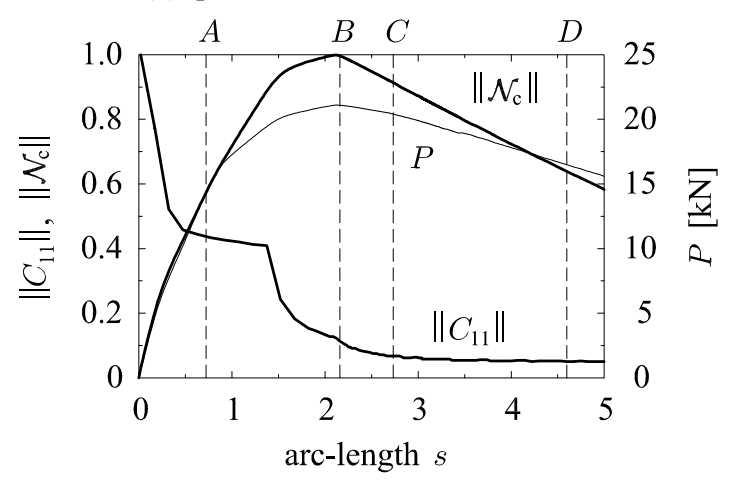

(c) point $T_{2}$

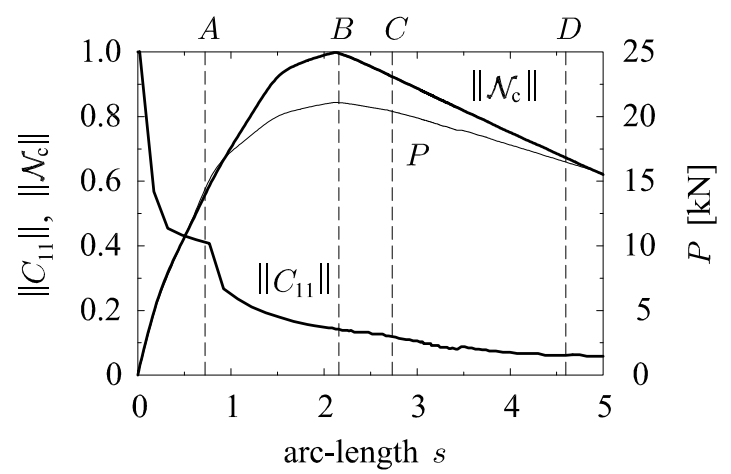

(b) point $T_{1}$

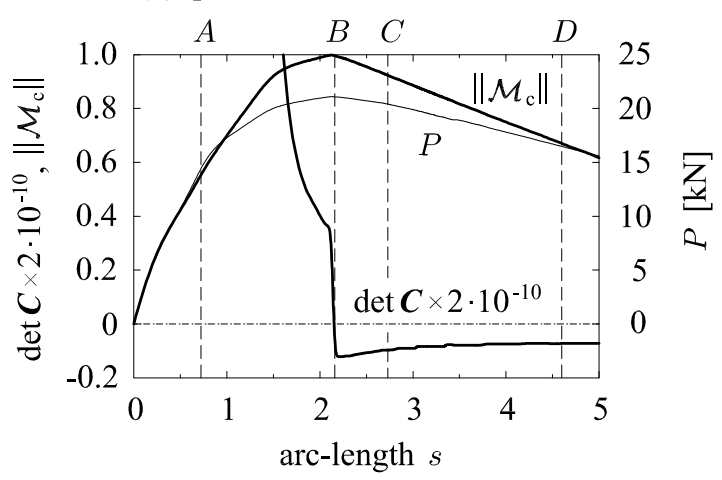

(d) point $T_{2}$

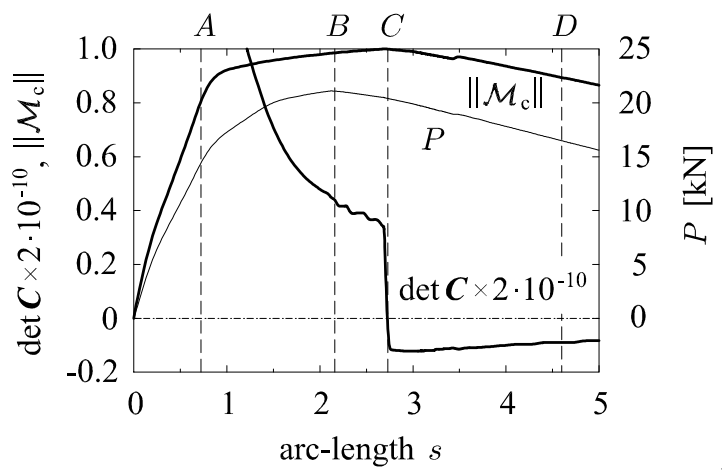

Figure 14. Cranston's portal frame. Graphs of $\mathcal{N}_{\mathrm{c}}, \mathcal{M}_{\mathrm{c}}, C_{11}$, and $\operatorname{det} \boldsymbol{C}$, as functions of arc-length $s$. (a)-(b) At the left-end cross-section of the beam, point $T_{1},(\mathrm{c})-(\mathrm{d})$ at the mid-point cross-section of the beam, point $T_{2}$. 
(a) Cross-section at point $T_{1}$

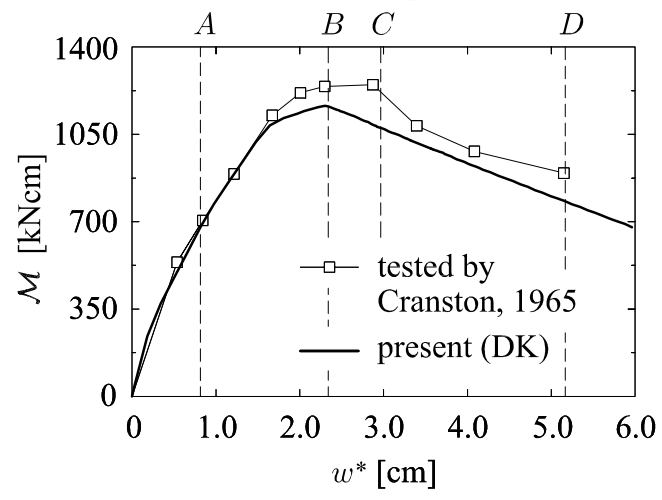

(b) Cross-section at point $T_{2}$

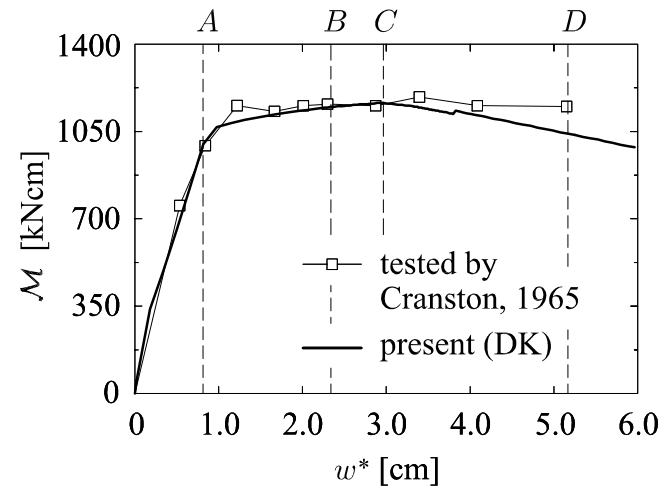

Figure 15. Cranston's portal frame. Bending moments. (a) At the left-end cross-section of the beam, point $T_{1}$, (b) at the mid-point cross-section of the beam, point $T_{2}$.

Point $C$ marks the state of the frame where the cross-section $T_{2}$ starts softening. The related force and mid-point deflection are $P_{C}=20.41 \mathrm{kN}$ and $w_{C}=2.97 \mathrm{~cm}$. The maximal compression strain in concrete at $T_{1}$ is $D_{\mathrm{c}}=-5.03 \%$, while the tension strain in steel bars is $D_{\mathrm{s}}=33.31 \%$, which is roughly three times larger than at the ultimate load, although it is still much smaller than the assumed ultimate strain $D_{\text {yu }}=300 \%$. Consequently, the constitutive moment at this cross-section has decreased and amounts to $\mathcal{M}=-1076 \mathrm{kNcm}$ (Fig. 15a). The decrease of the moment again indicates the softening of the cross-section. The determinant of the tangent constitutive matrix of the cross-section at $T_{1}$ is negative (see Fig. 14b). The maximal compression strain in concrete at the midpoint cross-section $T_{2}$ is $D_{\mathrm{c}}=-2.32 \%$. The tension strain in steel bars has increased to $D_{\mathrm{s}}=10.32 \%$. The bending moment at the cross-section has also increased and amounts to $\mathcal{M}=1164 \mathrm{kNcm}$ (Fig. $15 \mathrm{~b})$.

The redistribution of stresses in the beam during the softening phase is shown in Fig. 16. The Fig. 16a shows the normal stress distribution at $P_{A}=15.34 \mathrm{kN}$, and Fig. $16 \mathrm{~b}$ at $P_{D}=16.49 \mathrm{kN}$. It is indicated where the stresses are in the elastic regime (either in loading or unloading from the plastic state), or in the plastic regime (either in hardening or softening). At load $P_{A}$, the beam material is mostly elastic, and the bearing mechanism of the beam is a 'compression arch'. A relatively long region at the upper side of the beam at its central part is in the plastic regime. Once the global softening develops, the plastic region gets smaller and smaller due to the localization of deformation. Note also that load $P_{D}=16.49 \mathrm{kN}$ is greater than the load at $A$. 


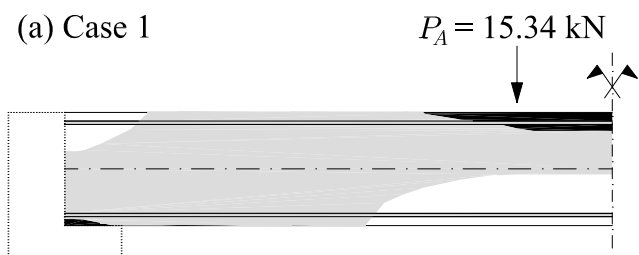

(c) Case 2

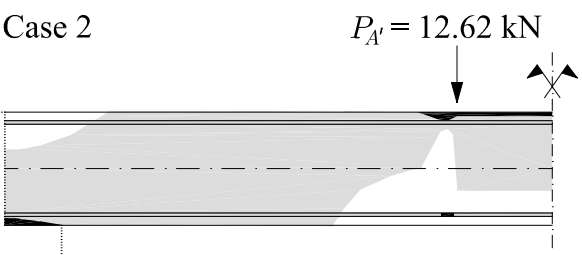

Stresses in concrete

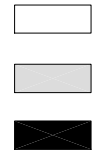

no stress

compression elastic regime

compression plastic regime (b) Case 1

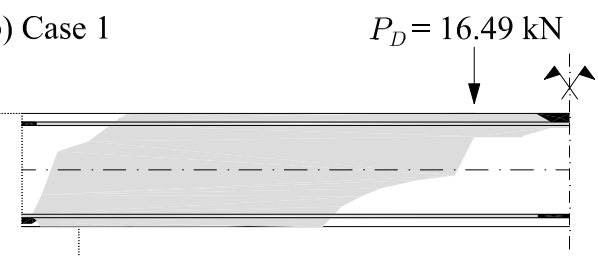

(d) Case 2

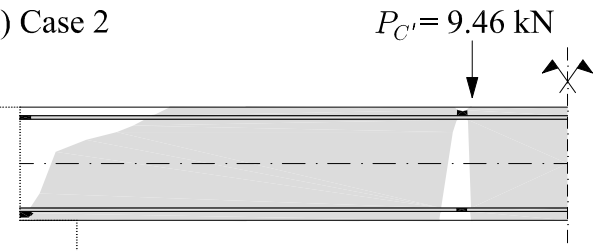

Stresses in steel elastic regime plastic regime

Figure 16. Cranston's portal frame. Stresses in the beam. (a) At load $P_{A}=15.34 \mathrm{kN}$ (point $A$ );

(b) at load $P_{D}=16.49 \mathrm{kN}$ (point $D$ ); (c) at load $P_{A^{\prime}}=12.62 \mathrm{kN}$ (point $A^{\prime}$ ); (d) at load

$$
P_{C^{\prime}}=9.46 \mathrm{kN}\left(\text { point } C^{\prime}\right) \text {. }
$$

We would like to show that only a minor change in the reinforcement length may cause major changes in the behaviour of the frame. We keep the material and geometrical data as in the previous analysis, and change only the length of the bars in the lower part of the cross-section, see Fig. 12b. Bažant et al. (1987a) assumed this reinforcement layout, yet they took different values of material parameters to model Cranston's frame.

We used the finite element mesh with 8 finite elements $E_{4-5}$ and 4 short constant-strain elements $E_{0-1}$ of length $\Delta L_{\mathrm{m}}=4 \mathrm{~cm}$. The mesh is depicted in Fig. $12 \mathrm{~b}$. This case will be referred to as 'Case 2' to distinguish it from the previous case, 'Case 1'.

The load-displacement curve for Case 2 is shown in Fig. 17 and compared to the results of Cranston's test and Case 1. The bearing capacity is now considerably lower. The sequence of the 'critical events' is also very different. This time the cross-section $T_{3}$ and its symmetric companion $T_{3}^{\prime}$ (see Fig. 12b) first reach their ultimate bearing capacities at load $P_{A^{\prime}}=12.62 \mathrm{kN}$ ( $A^{\prime}$ is the point on the load-displacement curve, see Fig. 17) which is smaller than the ultimate critical load of the frame $\left(P_{B^{\prime}}=15.52 \mathrm{kN}\right)$. This is due to the fact, that the cross-section $T_{3}$ is insufficiently reinforced to compensate fully 
the imposed (the equilibrium) bending moment. During the subsequent deformation, the cross-sections $T_{3}$ and $T_{3}^{\prime}$ soften. At point $B^{\prime}$ the bearing capacity of cross-section $T_{1}$ and its symmetric companion $T_{1}^{\prime}$, and of the frame are reached simultaneously. Since then the cross-sections $T_{1}, T_{1}^{\prime}, T_{3}$ and $T_{3}^{\prime}$ soften. Let us recall that Bažant et al. (1987a) employed the reinforcement layout of Case 2. Their load-displacement curve is displayed in Fig. 13 and a good agreement may be observed with Cranston's. Yet material parameters they employed do not fully agree with those measured in Cranston's tests. For example, their yield strength of steel was assumed to be $f_{\mathrm{y}}=40 \mathrm{kN} / \mathrm{cm}^{2}$ instead of $f_{\mathrm{y}}=29.3 \mathrm{kN} / \mathrm{cm}^{2}$ given by Cranston. Such a change of material data influences the results substantially and offers possibilities to fit the numerical response curve with the one obtained in the test.

Fig. 16 shows the no-stress, elastic, and plastic regions in concrete and steel for Case 2. The figure again confirms that a small change in the reinforcement layout causes notably different distributions of stresses in the frame. This is also true for the deformed shapes of the two frames in the softening phase (Fig. 18). The differences between the deformed shapes are clearly seen.

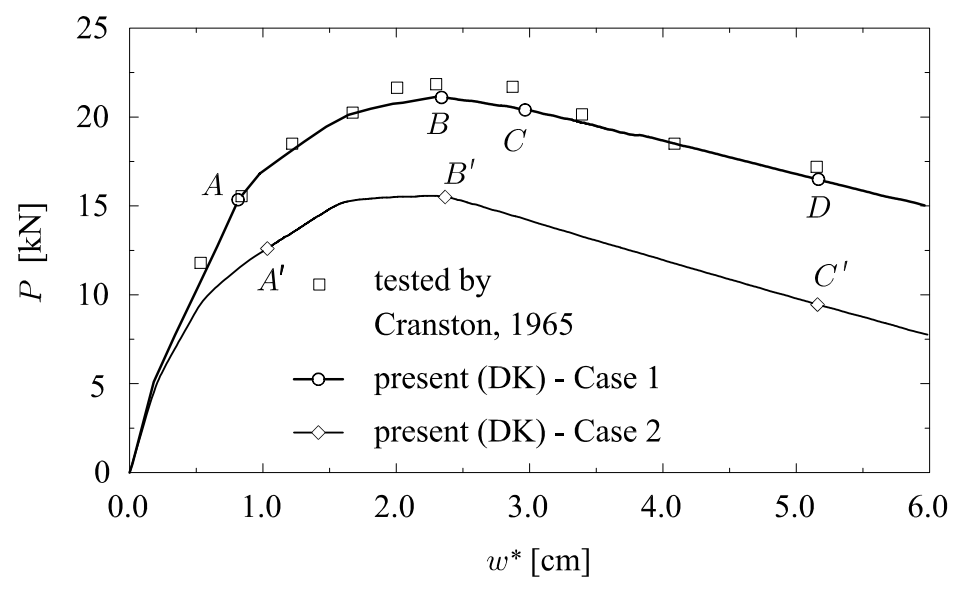

Figure 17. Cranston's portal frame. The load-displacement curve as a function of the reinforcement layout, Case 1 and Case 2.

Our analyses assumed a complete symmetry of the frame. This was also assumed by Lazaro and Richards (1973) and Bažant et al. (1987a). In Cranston's test, however, a large horizontal displacement was reported and attributed to an initial geometrical imperfection of the frame. Therefore, we also analysed the frame (see Fig. 20b) with the skew columns, 
(a) Case 1

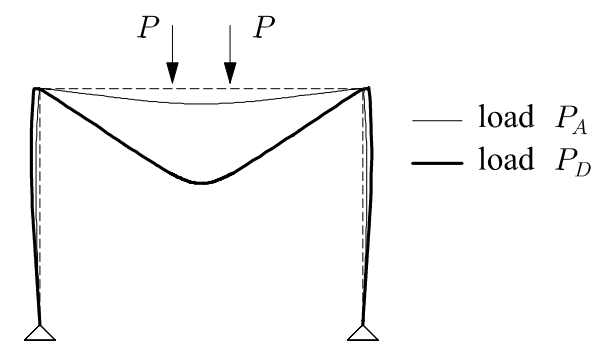

(b) Case 2

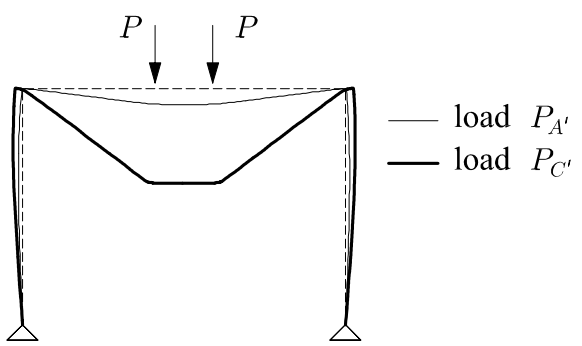

Figure 18. Cranston's portal frame. The comparison of deformed shapes in the softening phase (magnified $15 \times)$.

the small imperfection being $\Delta=0.32 \mathrm{~cm}$. To fit the measured response, we had to change the following data: $D_{\mathrm{y} 2}=11 \%$ (instead of $10 \%$ taken previously); $D_{\mathrm{yu}}=1300 \%$ $(300 \%) ; D_{\text {cu }}=-100 \%(-50 \%) ; G_{f}^{\mathrm{c}}=25 \mathrm{~N} / \mathrm{mm}(20 \mathrm{~N} / \mathrm{mm})$, while the rest of the material data remains as in Case 1. The finite-element mesh, consisting of 15 short elements $\left(\Delta L_{\mathrm{m}}=4 \mathrm{~cm}\right)$ and eight $E_{4-5}$ elements, is displayed in Fig. 19.

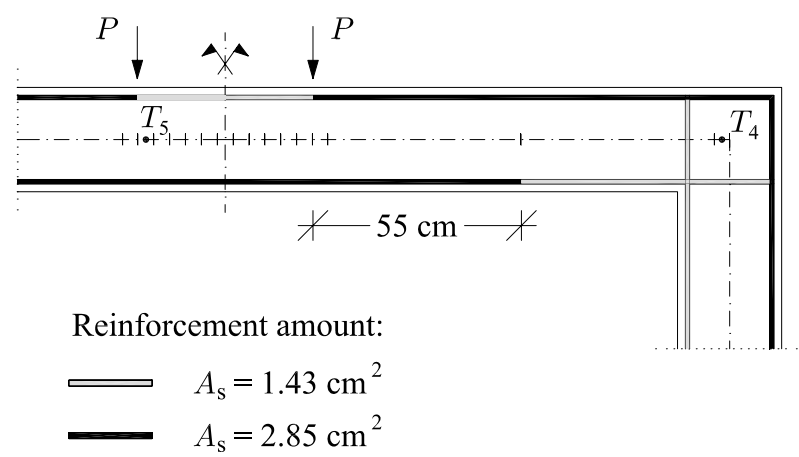

Figure 19. Cranston's portal frame with a geometric imperfection. Finite-element mesh.

Fig. 20a shows the load-displacement curves of the imperfect frame. The vertical displacement $w^{*}$ at point $T_{2}$ and the horizontal displacement $u^{*}$ at point $T_{1}$ are displayed. The comparison with the results of the test shows excellent agreement between the computed and experimental results.

At point $F$ of the load-displacement curve the frame reaches the ultimate critical load $P_{\text {cr }}=21.09 \mathrm{kN}$. Simultaneously, the cross-section at $T_{4}$ (see Fig. 19) reaches the ultimate bearing capacity. The related compressive strain in concrete at the bottom of 
the cross-section is $D_{\mathrm{c}}=-2.59 \%$ o , and the tension strain in the steel bar at the top of the cross-section is $D_{\mathrm{s}}=12.36 \%$. It appears that the ultimate critical load of the frame is only moderately sensitive to the geometrical imperfection. The vertical displacement is roughly the same, too: $w_{\mathrm{cr}}^{*}=2.27 \mathrm{~cm}$ compared to $2.34 \mathrm{~cm}$ in the perfect frame. The horizontal displacement, however, is now four times greater: $u_{\mathrm{cr}}^{*}=0.42 \mathrm{~cm}$ compared to $0.11 \mathrm{~cm}$ in the perfect frame. The values are in a very good accord with the measured ones (Fig. 20a).

(a) Load-displacement curves

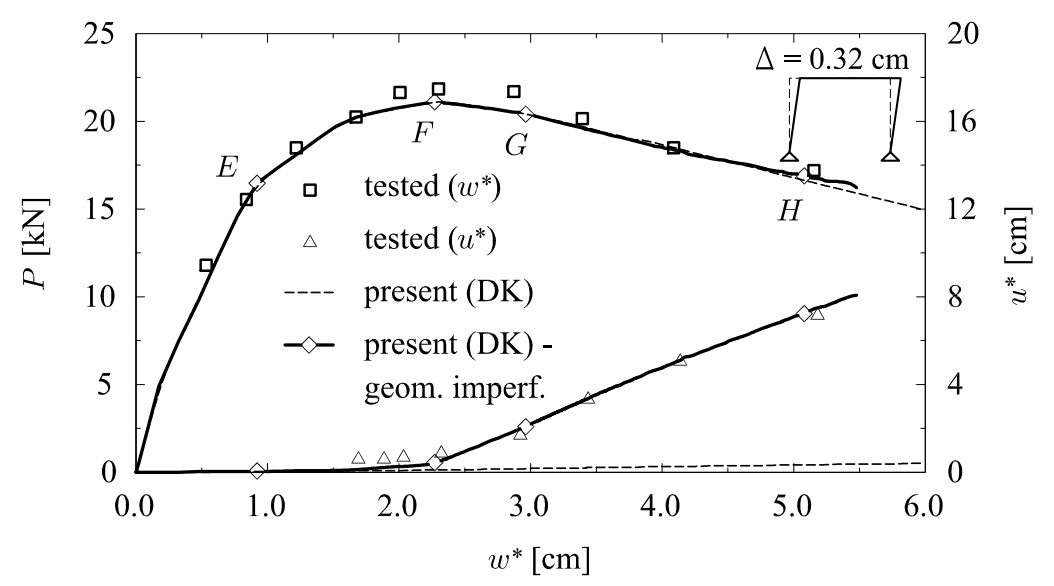

(b) Deformed shapes
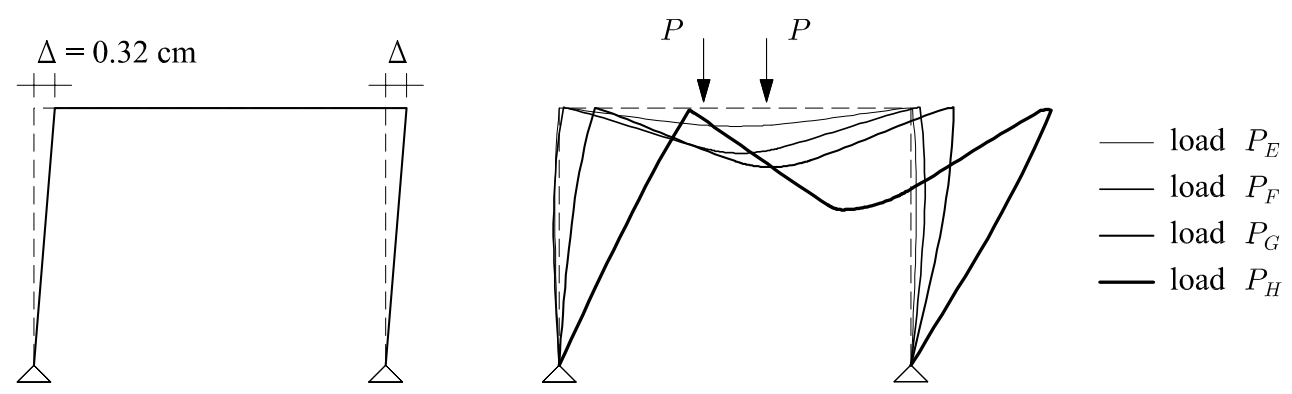

Figure 20. Cranston's portal frame with the geometric imperfection. (a) Load-displacement curves; (b) deformed shapes in softening regime (15× magnified).

At $P_{G}=20.42 \mathrm{kN}$ (the corresponding vertical displacement is $w_{G}^{*}=2.97 \mathrm{~cm}$ ) the crosssection $T_{5}$ (see Fig. 19) starts softening. This compares well with the situation without the imperfection (see point $C$ in Fig. 13), only that now the horizontal displacement of point $T_{5}$ is $u_{G}^{*}=2.09 \mathrm{~cm}$, which is about 12 times larger than the displacement of the perfect frame $\left(u_{G}^{*}=0.17 \mathrm{~cm}\right)$. 
The deformed shapes of the frame in the post-critical softening phase are shown in Fig. 20b. Observe that the displacements in Fig. 20b are 15 times magnified.

In Fig. 21 we display the distributions of the extensional and bending strains along the axis of the frame for two deformation stages in the softening phase of the frame. The graphs clearly show a very high localization of deformations at points $T_{4}$ and $T_{5}$. We wish to stress that the inclusion of constant-strain elements in the finite-element mesh was essential to trigger the localization of deformation. We note that both strain measures, $\varepsilon$ and $\kappa$, have localized. This shows how important it is to consider both strain measures in the analyses. Many beam formulations (e.g. Bažant et al., 1987a; Jirásek, 1997) neglect extensional strain in the strain-softening calculations, which often leads to unsatisfactory results.

(a) Extensional strain $\varepsilon$ at load $P_{F}$

(b) Bending strain $\kappa$ at load $P_{F}$
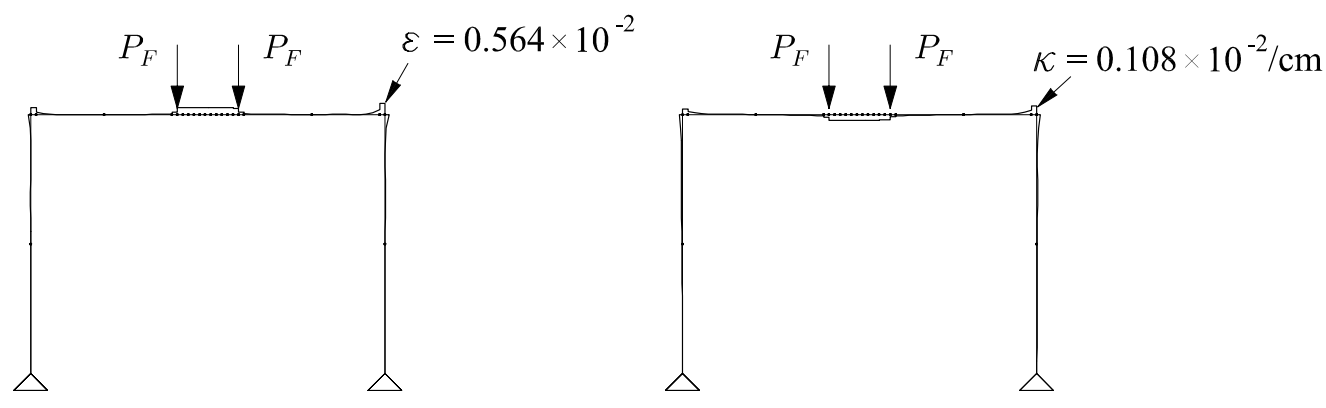

(c) Extensional strain $\varepsilon$ at load $P_{H}$

(d) Bending strain $\kappa$ at load $P_{H}$

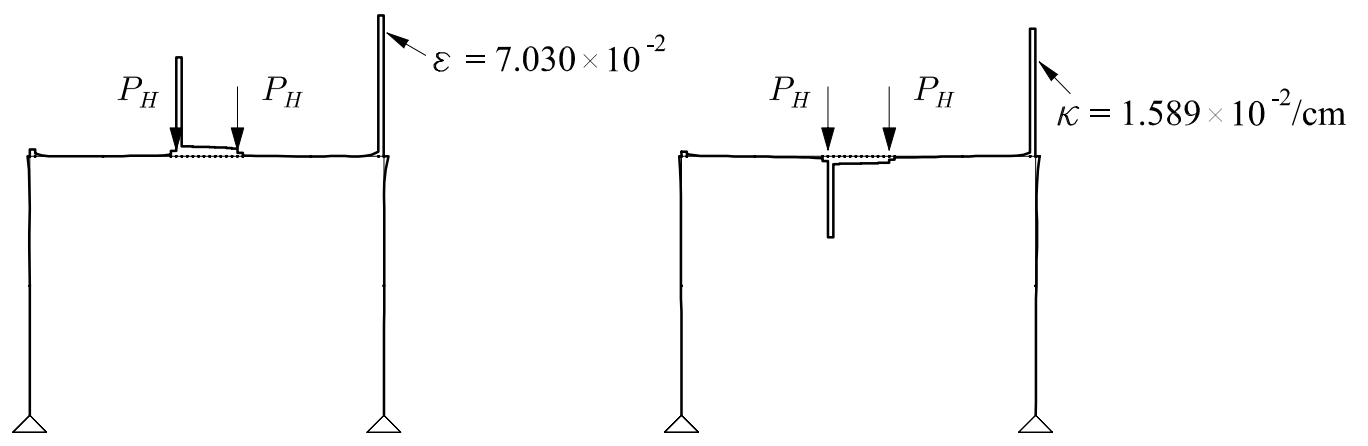

Figure 21. Cranston's portal frame with the geometric imperfection. The distribution of strain measures, $\varepsilon$ and $\kappa$, along the axis of the frame. (a) $\varepsilon$ at $P_{F}$, (b) $\kappa$ at $P_{F}$, (c) $\varepsilon$ at $P_{H}$, (d) $\kappa$ at $P_{H}$.

Our final remark concerns the numerical integration over the cross-section. Fig. 22 shows the distributions of the normal stress and the tangent modulus in concrete at cross- 
sections $T_{1}$ and $T_{2}$ at $P=0.93 P_{\mathrm{cr}}=20 \mathrm{kN}$ for the geometrically perfect frame, Case 1 . It is clear that the stress and the tangent modulus are discontinuous functions of $z$. Because standard Gaussian integrations require that the integrated functions are continuous, these methods are not directly applicable in such situations. Fig. 23 shows the convergence graphs for four characteristic quantities of the cross-section $T_{1}: \mathcal{N}_{\mathrm{c}}, \mathcal{M}_{\mathrm{c}}$, and $C_{11, \mathrm{c}}$ in concrete, and $\operatorname{det} \boldsymbol{C}_{\mathrm{c}}$ of the concrete part of the cross-section. 1, 3, 5, 10, 20, and 50 layers are used, respectively, and 10-point Gaussian or Lobatto's integration within each layer to model the cross-section. Gaussian integration results in much more accurate results for small number of layers ( 1 or 3 ), while for a bigger number of layers, the two integration methods become comparable. The increase of the number of layers seems to improve the results. The convergence, however, is relatively slow (see Fig. 23b). This is due to the discontinuous distribution of stresses and material tangent moduli over the cross-section. A special numerical integration technique seems to be required to improve the results.

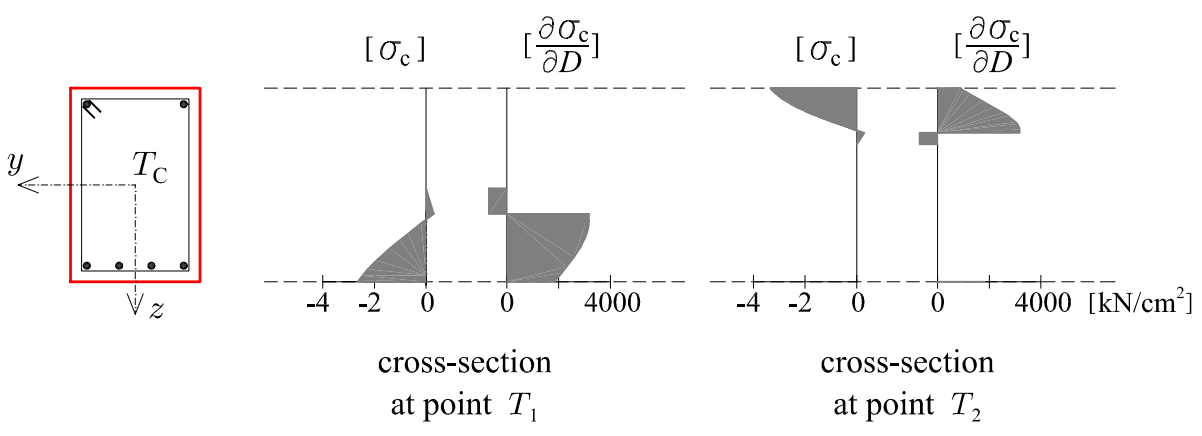

Figure 22. Cranston's portal frame. The distributions of normal stress and tangent modulus in concrete at cross-sections $T_{1}$ and $T_{2}$ at $P=20 \mathrm{kN}$.

\section{Conclusions}

The materially and geometrically non-linear analysis of concrete structures is a difficult task. The analysis tool must be sophisticated enough to capture the phenomena like strain-softening and strain localization in concrete with a sufficient precision. In order to construct such a tool, we have derived a new family of beam finite elements. They are based on Reissner's planar beam theory and therefore consider exact geometrical nonlinearity. Their novelties are: (i) the extensional and the bending strains are the only interpolated functions; (ii) the equilibrium and the constitutive internal forces are equal at 
(a)

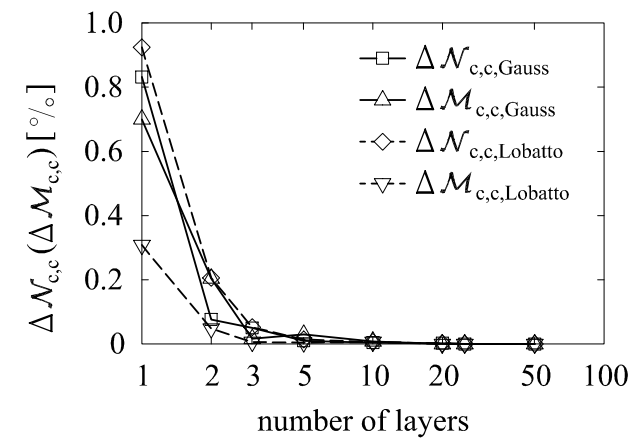

(b)

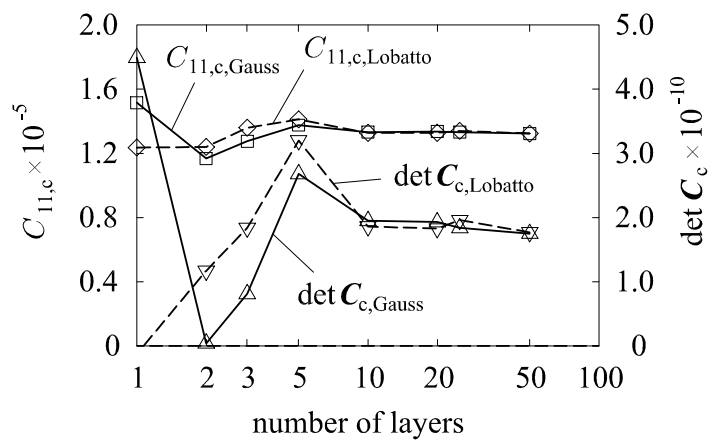

Figure 23. Cranston's portal frame. The accuracy of numerical integrations over the cross-section at $T_{1} ; P=20 \mathrm{kN}$. (a) $\Delta \mathcal{N}_{\mathrm{c}, \mathrm{c}}\left(\Delta \mathcal{M}_{\mathrm{c}, \mathrm{c}}\right)=\left|\frac{\mathcal{N}(\mathcal{M})_{\mathrm{c}}-\mathcal{N}(\mathcal{M})_{c, 50}}{\mathcal{N}(\mathcal{M})_{\mathrm{c}, 50}}\right|$ in concrete, (b) $C_{11, \mathrm{c}}$ in concrete, and $\operatorname{det} \boldsymbol{C}_{\mathrm{c}}$ of the concrete part of the cross-section.

the integration points. The members of the finite element family can equally well describe extensible or inextensible beams, shear stiff or shear deformable beams, and thin or thick beams. The elements are rigid-body displacement invariant and path-independent for conservative problems. The elements are applied in the analysis of the reinforced concrete planar frames.

We employed the EC 2 constitutive model of concrete and the model of Desayi and Krishnan (1964). The strain-hardening as well as the strain-softening of concrete were taken into account, yet, for the present, the effect of creep and shrinkage in concrete were neglected. The three-linear stress-strain diagram for the reinforcing steel was assumed along with the isotropic-type of the strain-hardening and softening. The no-slip contact between concrete and steel was assumed.

The efficiency of our finite element model was proved through the analyses of three realistic frame structures. All of them were previously tested in the laboratory. The behaviour of the first structure is characterized by the dominance of the geometric instability, whereas the behaviour of the third structure is more materially dominant. We directed our analyses into the study of the post-critical behaviour of frames, in particular into the description of the material softening of cross-sections, the softening of the global response, and their interaction. The results include the graphs of the strain localizations at cross-sections and the redistributions of regions of strain-hardening, strain-softening and unloading. 
Fundamental to our strain-softening analysis is the introduction of the short constantstrain element in our new beam finite element family. This enables us to detect automatically the loss of the local stability at the cross-section and to proceed into the strain-softening regime.

\section{References}

Bathe, K.J., 1996. Finite Element Procedures. Prentice-Hall, Inc., Simon \& Schuster, New Jersey.

Bažant, Z., Pan, J., Pijaudier-Cabot, G., 1987a. Softening in reinforced concrete beams or frames. ASCE Journal of Structural Engineering 113, 2333-2347.

Bažant, Z., Pijaudier-Cabot, G., Pan, J., 1987b. Ductility, snapback, size effect, and redistribution in softening beams or frames. ASCE Journal of Structural Engineering 113, 2348-2364.

Bažant, Z., Mazars, J., 1990. France-U.S. workshop on strain localization and size effect due to cracking and damage. ASCE Journal of Engineering Mechanics 116, 1412-1424.

Bergan, P.G., Holand, I., 1979. Nonlinear finite element analysis of concrete structures. Computer Methods in Applied Mechanics and Engineering 17/18, 443-467.

Carol, I., Murcia, J., 1989. Nonlinear time-depending analysis of planar frames using an 'exact' formulation - II. Computer implementation for r.c. structures and examples. Computers and Structures 33, 89-102.

Chen, G., Baker, G., Hunt, G.W., 2000. Computational approach to localization using global energy minimization. Computers and Structures 78, 529-536.

Coleman, J., Spacone, E., 2001. Localization issues in force-based frame elements. ASCE Journal of Structural Engineering 127, 1257-1265.

Contrafatto, L., Cuomo, M., 2002. A new thermodynamical consistent continuum model for hardening plasticity coupled with damage. International Journal of Solids and Structures 39, $6241-6271$.

Cowper, G.R., 1966. The shear coefficient in Timoshenko's beam theory. ASME Journal of Applied Mechanics 33, 335-340.

Cranston, W.B., 1965. Tests on reinforced concrete frames, 1: Pinned portal frames. Technical Report TRA/392, Cement and Concrete Association, London, England.

Crisfield, M.A., 1981. A fast incremental/iterative solution procedure that handles 'snap-through'. Computers and Structures 13, 55-62.

Crisfield, M.A., 1991, 1997. Non-Linear Finite Element Analysis of Solids and Structures. Volumes 1 and 2, John Wiley \& Sons, Chichester. 
Darvall, P.L., Mendis, P.A., 1985. Elastic-plastic-softening analysis of plane frames. ASCE Journal of Structural Engineering 111, 871-888.

Desayi, P., Krishnan, S., 1964. Equation for the stress-strain curve of concrete. Journal of American Concrete Institute 61, 345-350.

El-Metwally, S.E., El-Shahhat, A.M., Chen, W.F., 1990. 3D nonlinear analysis of R/C slender columns. Computers and Structures 37, 863-872.

Espion, B., 1993. Benchmark examples for creep and shrinkage analysis computer programs, Creep and shrinkage of concrete. TC 114 RILEM. E\&FN Spon.

Eurocode 2: Design of concrete structures, 1999. Part 1: General rules and rules for buildings, prEN 1992-1: 2001 ( $1^{\text {st }}$ draft).

Feng, Y.T., Perić, D., Owen, D.R.J., 1996. A new criterion for determination of initial loading parameter in arc-length methods. Computers and Structures 58, 479-485.

Ferguson, P.M., Breen, J.E., 1966. Investigation of the long concrete column in a frame subjected to lateral loads. Symposium on Reinforced Concrete Columns, American Concrete Institute SP-13.

Grassel, P., Lundgren, K., Gylltoft, K., 2002. Concrete in compression: a plasticity theory with a novel hardening law. International Journal of Solids and Structures 39, 5205-5223.

Gunnin, B.L., Rad, F.N., Furlong, R.W., 1977. A general nonlinear analysis of concrete structures and comparison with frame tests. Computers and Structures 7, 257-265.

Jansen, D.C., Shah, S.P., 1997. Effect of length on compressive strain-softening of concrete. ASCE Journal of Engineering Mechanics 123, 25-35.

Jelenić, G., Saje, M., 1995. A kinematically exact space finite strain beam model-Finite element formulation by generalized virtual work principle. Computer Methods in Applied Mechanics and Engineering 120, 131-161.

Jirásek, M., 1997. Analytical and numerical solutions for frames with softening hinges. ASCE Journal of Engineering Mechanics 123, 8-14.

Khaloo, A.R., Tariverdilo, S., 2002. Localization analysis of reinforced concrete members with softening behavior. ASCE Journal of Structural Engineering 128, 1148-1157.

Kim, J.K., Lee, T.G., 1992. Nonlinear analysis of reinforced concrete beams with softening. Computers and Structures 44, 567-573.

Kwak, H.-K., Kim, S.-P., 2002. Nonlinear analysis of RC beams based on moment-curvature relation. Computers and Structures 80, 615-628. 
Lazaro, A.L., Richards, R., 1973. Full-range analysis of concrete frames. ASCE Journal of the Structural Division 99, 1761-1783.

Maier, M., Perego, U., 1992. Effects of softening in elastic-plastic structural dynamics. International Journal for Numerical Methods in Engineering 34, 319-347.

Mendis, P.A., Darvall, P.L., 1988, Stability analysis of softening frames. ASCE Journal of Structural Engineering 114, 1057-1072.

Peerlings, R.H.J., de Borst, R., Brekelmans, W.A.M., Geers, M.G.D., 2002, Localization issues in local and nonlocal continuum approaches to fracture. European Journal of Mechanics A/Solids 21, 175-189.

Planinc, I., Saje, M., Čas, B., 2001. On the local stability condition in the planar beam finite element. Structural Engineering and Mechanics 12, 507-526.

Pöttier, R., Swoboda, G.A., 1987. Nonlinear beam element for r/c structures. Communications in Applied Numerical Methods 3, 397-406.

Rasheed, H.A.S., Dinno, K.S., 1994. An improved nonlinear analysis of reinforced concrete frames. Computers and Structures 53, 625-636.

Reissner, E., 1972. On one-dimensional finite-strain beam theory: the plane problem. Journal of Applied Mathematics and Physics (ZAMP) 23, 795-804.

Saje, M., 1990. A variational principle for finite planar deformation of slender elastic frames. International Journal of Solids and Structures 26, 887-900.

Saje, M., Planinc, I., Turk, G., Vratanar, B., 1997. A kinematically exact finite element formulation of planar elastic-plastic frames. Computer Methods in Applied Mechanics and Engineering 144, 125-151.

Simo, J.C., Hjelmstad, K.D, Taylor, R.L., 1984. Numerical formulations for finite deformation problems of beams accounting for the effect of transverse shear. Computer Methods in Applied Mechanics and Engineering 42, 301-330.

Simo, J.C., 1985. A finite strain beam formulation. The three-dimensional dynamic problem. Part I. Computer Methods in Applied Mechanics and Engineering 49, 55-70.

Stolarski, H., Belytschko, T., 1983. Shear and membrane locking in curved Co elements. Computer Methods in Applied Mechanics and Engineering 41, 279-296.

Vratanar, B., Saje, M., 1998. A consistent equilibrium in a cross-section of an elastic-plastic beam. International Journal of Solids and Structures 36, 311-337.

Wells, G.N., Sluys, L.J., 2000. Application of embedded discontinuities for softening solids. Engineering Fracture Mechanics 65, 263-281. 